\title{
TWISTS OF HOOLEY'S $\triangle$-FUNCTION OVER NUMBER FIELDS
}

\author{
EFTHYMIOS SOFOS
}

\begin{abstract}
We prove tight estimates for averages of the twisted Hooley $\Delta$-function over arbitrary number fields.
\end{abstract}

\section{INTRODUCTION}

In his memoir [Hoo79] Hooley studied the following function, previously brought to attention by Erdős,

$$
\Delta(n):=\max _{a \in \mathbb{R}} \sharp\left\{d \in \mathbb{N}: \mathrm{e}^{a}<d \leqslant \mathrm{e}^{a+1}, d \mid n\right\}, \quad(n \in \mathbb{N}) .
$$

He showed that its average order is genuinely smaller than that of the divisor function, namely

$$
\frac{1}{x} \sum_{n \leqslant x} \Delta(n) \ll(\log x)^{\frac{4}{\pi}-1} .
$$

This saving enabled him to provide diverse applications in topics related to Diophantine approximation, divisor sums and problems of Waring's type. Further applications were later found by Vaughan [Vau85], Vau86], for problems of Waring's type, by Tenenbaum [Ten86] in the topic of Diophantine approximation, as well as for Chebychev's problem on the greatest prime factor of polynomial sequences by Tenenbaum [Ten90].

The problem regarding the average of $\Delta$ was revisited by Tenenbaum [Ten85], who established a strong upper bound, with a special corollary that the exponent $\frac{4}{\pi}-1$ can be replaced by any positive constant. Specifically, letting

$$
\hat{\varepsilon}(x)=\sqrt{\frac{\log \log \log (16+x)}{\log \log (3+x)},}
$$

for any $x \geqslant 1$, enables us to state his result, namely

$$
\frac{1}{x} \sum_{n \leqslant x} \Delta(n) \ll(\log x)^{O(\widehat{\varepsilon}(x))} .
$$

In this paper we are interested in generalisations of functions similar to $\Delta$ over arbitrary number fields. Let $K$ be any number field with ring of integers denoted by $\mathscr{O}_{K}$. The symbol $\mathscr{I}_{K}$ will be reserved for the monoid of non-zero integral ideals of $\mathscr{O}_{K}$, while $\mathfrak{N a}=\sharp \mathscr{O}_{K} / \mathfrak{a}$ will always refer to the ideal norm of $\mathfrak{a} \in \mathscr{I}_{K}$. The generalisation of (1.1) to $K$ is given by

$$
\Delta_{K}(\mathfrak{a}):=\max _{a \in \mathbb{R}} \sharp\left\{\mathfrak{d} \in \mathscr{I}_{K}: \mathrm{e}^{a}<\mathfrak{N} \mathfrak{d} \leqslant \mathrm{e}^{a+1}, \mathfrak{d} \mid \mathfrak{a}\right\}, \quad\left(\mathfrak{a} \in \mathscr{I}_{K}\right) .
$$

The following result and its proof are entirely due to Professors Régis de la Bretèche and Gérald Tenenbaum dlBT16. 
Theorem 1.1. There exists a positive constant $c=c(K)$ such that

$$
\frac{1}{x} \sum_{\mathfrak{N a} \leqslant x} \Delta_{K}(\mathfrak{a}) \ll(\log x)^{c \hat{\varepsilon}(x)},
$$

where the implied constant is allowed to depend on $K$.

To show Theorem 1.1, De la Bretèche and Tenenbaum begin by showing that

$$
\frac{1}{x} \sum_{\mathfrak{N a} \leqslant x} \Delta_{K}(\mathfrak{a}) \ll \frac{1}{\log x} \sum_{n \leqslant x} \frac{\mu(n)^{2}}{n} \sum_{\mathfrak{N} \mathfrak{a}=n} \Delta_{K}(\mathfrak{a})
$$

in a way that is familiar to experts (see, for example [NT98]). They now observe that for square-free $n$, if there exists $d \in \mathbb{N}$ with $d \mid \mathfrak{N a}$ then there exists a unique ideal $\mathfrak{d}$ with $\mathfrak{d} \mid n$, thus implying that $\Delta_{K}(\mathfrak{a})=\Delta(n)$ in the last sum. This shows that

$$
\frac{1}{x} \sum_{\mathfrak{N} \mathfrak{a} \leqslant x} \Delta_{K}(\mathfrak{a}) \ll \frac{1}{\log x} \sum_{n \leqslant x} \frac{\mu(n)^{2}}{n} r_{K}(n) \Delta(n),
$$

where

$$
r_{K}(n):=\sharp\left\{\mathfrak{d} \in \mathscr{I}_{K}: \mathfrak{N d}=n\right\}, \quad(n \in \mathbb{N}) .
$$

Next, choosing a monic irreducible polynomial $f \in \mathbb{Z}[X]$ such that $K=\mathbb{Q}(\theta)$ for a root $\theta$ of $f$, one can use the Dedekind-Kummer theorem to relate the function $r_{K}$ and $\varrho_{f}$, defined by

$$
\varrho_{f}(n):=\sharp\{t \in \mathbb{Z} / n \mathbb{Z}: f(t) \equiv 0(\bmod n)\},
$$

in particular, getting

$$
\sum_{n \leqslant x} \frac{\mu(n)^{2}}{n} r_{K}(n) \Delta(n) \ll \sum_{n \leqslant x} \frac{\mu(n)^{2}}{n} \varrho_{f}(n) \Delta(n) .
$$

As a last step, they applied [Ten90, Th.3] to establish Theorem 1.1, and, in addition, they showed that one can take any fixed constant $c>\sqrt{2}$.

Next, let $\psi_{K}$ be any quadratic Dirichlet character on $K$ and define

$$
\Delta_{K}\left(\mathfrak{a} ; \psi_{K}\right):=\sup _{\substack{a \in \mathbb{R} \\ 0 \leqslant b \leqslant 1}}\left|\sum_{\substack{\mathfrak{d} \mid \mathfrak{a} \\ \mathrm{e}^{a}<\mathfrak{N} \mathfrak{d} \leqslant \mathrm{e}^{a+b}}} \psi_{K}(\mathfrak{d})\right|, \quad\left(\mathfrak{a} \in \mathscr{I}_{K}\right) .
$$

When $K=\mathbb{Q}$ this function was considered by La Bretèche and Tenenbaum dlBT12, as well as by Brüdern [Brü12. Their work culminates in the bound,

$$
\frac{1}{x} \sum_{n \leqslant x} \Delta_{\mathbb{Q}}\left(n ; \psi_{\mathbb{Q}}\right)^{2} \ll(\log x)^{O(\widehat{\varepsilon}(x))} .
$$

In this paper we generalise this to any number field by following the arguments in dlBT12.

Theorem 1.2. Let $\psi_{K}$ be a quadratic Dirichlet character defined on any number field $K$. There exists a positive constant $c=c\left(K, \psi_{K}\right)$ such that

$$
\frac{1}{x} \sum_{\mathfrak{N a} \leqslant x} \Delta_{K}\left(\mathfrak{a} ; \psi_{K}\right)^{2} \ll(\log x)^{c \hat{\varepsilon}(x)},
$$

where the implied constant is allowed to depend on $K$ and $\psi_{K}$. 
Professors Régis de la Bretèche and Gérald Tenenbaum have informed us that there may be a neater way to prove Theorem 1.2 without simply translating their work dlBT12 to number fields, which is what we do in the present paper. It must be noted that one cannot directly deduce Theorem 1.2 for all number fields $K$ from the work in dlBT12 in the same way that Theorem 1.1 was deduced from [Ten90, Th.3]. This direct deduction can only be made if $K$ is such that there exists a Dirichlet character $\chi$ defined in $\mathbb{Q}$ and satisfying

$$
\mathfrak{a} \in \mathscr{I}_{K} \Rightarrow \psi_{K}(\mathfrak{a})=\chi(\mathfrak{N a}) .
$$

However, (1.4) does not hold for every $K$ and $\psi_{K}$. Indeed, let $K / \mathbb{Q}$ be a quadratic extension, let $L / K$ be a quadratic extension and define a quadratic character $\psi_{K}$ by defining $\psi_{K}(\mathfrak{p})$ to be $1,-1$ or 0 respectively according to whether $\mathfrak{p}$ is split, inert, or ramifies in $L$. If $p$ is a rational prime such that $p \mathscr{O}_{L}$ is inert in $L$ and (1.4) holds, then

$$
-1=\psi_{K}(\mathfrak{p})=\chi(\mathfrak{N p})=\chi\left(p^{2}\right)=1,
$$

which gives a contradiction. We also provide a number field $K$ for which the following relaxed version of (1.4) fails: assume that there exists a function $g: \mathbb{Z} \rightarrow \mathbb{C}$ such that

$$
\mathfrak{p} \in \mathscr{I}_{K}, \mathfrak{N p} \text { is a prime in } \mathbb{Z} \Rightarrow \psi_{K}(\mathfrak{p})=g(\mathfrak{N p})
$$

Take

$$
K:=\mathbb{Q}(\sqrt{-1}) \text { and } \psi_{K}(\mathfrak{a}):=\left(\frac{1+\sqrt{-1}}{\mathfrak{a}}\right),
$$

where $\left(\frac{1+\sqrt{-1}}{\cdot}\right)$ is a quadratic symbol in $K$. For every rational prime $p$ with $p \equiv 5(\bmod 8)$ we have $p \mathscr{O}_{K}=\mathfrak{p} \overline{\mathfrak{p}}$ for a prime ideal $\mathfrak{p}$ in $\mathscr{I}_{K}$. Letting $\pm t$ be the two solutions of $t^{2} \equiv-1(\bmod p)$, the Dedekind-Kummer theorem shows that

$$
\left\{\psi_{K}(\mathfrak{p}), \psi_{K}(\overline{\mathfrak{p}})\right\}=\left\{\left(\frac{1+t}{p}\right),\left(\frac{1-t}{p}\right)\right\},
$$

where $(\dot{\bar{p}})$ is the Legendre quadratic symbol in $\mathbb{F}_{p}$. Note that $\mathfrak{N p}=\mathfrak{N} \bar{p}$ is a prime in $\mathbb{Z}$, therefore, if (1.5) holds, then $\psi_{K}(\mathfrak{p})=\psi_{K}(\overline{\mathfrak{p}})$. This means that

$$
1=\psi_{K}(\mathfrak{p}) \psi_{K}(\overline{\mathfrak{p}})=\left(\frac{1+t}{p}\right)\left(\frac{1-t}{p}\right)=\left(\frac{1+t^{2}}{p}\right)=\left(\frac{2}{p}\right) .
$$

Recall that $p \equiv 5(\bmod 8)$, therefore $\left(\frac{2}{p}\right)=-1$. This shows that (1.5) cannot hold for our $K$.

The proof of Theorem 1.2, supplied in $₫ 3$, follows closely the approach in [lBT12] involving an induction related to the number of prime ideal divisors of $\mathfrak{a}$. There will only be minor modifications; these are to take care of the fact that there may be several ideals $\mathfrak{d}$ of a given norm in (1.2). Thus one has to deal with short sums $\sum_{\mathfrak{d}} \psi_{K}(\mathfrak{d})$ that contain an amount of terms which is not necessarily bounded. We have to show that cancellation in this short sum still occurs in this situation and it will turn out that the arguments of La Bretèche and Tenenbaum dIBT12] are flexible enough to handle these issues when suitably modified.

Let us finally remark that interest in averages of $\Delta$-functions has lately spiked due to applications to Manin's conjecture. This is a central conjecture in the area of Diophantine geometry, introduced by Manin and his collaborators in [FMT89], whose aim is to provide a precise description of the distribution of rational points on Fano varieties. However, its status 
for surfaces has not yet been fully resolved. An important rôle in proving the conjecture for Châtelet surfaces is assumed by the asymptotic estimation of divisor sums of the form

$$
\sum_{\substack{(s, t) \in \mathbb{Z}^{2} \\|s|,|t| \leqslant x}} \sum_{\substack{d \in \mathbb{N} \\ d \mid F(s, t)}} \psi_{\mathbb{Q}}(d),
$$

as $x \rightarrow+\infty$, where $F \in \mathbb{Z}[s, t]$ is a separable quartic form and $\psi_{\mathbb{Q}}$ is a quadratic Dirichlet character. La Bretèche and Tenenbaum used (1.3) to handle these divisor sums when $F$ is irreducible or a product of two irreducible quadratic forms in dlBT13, which enabled them to prove Manin's conjecture for two families of Châtelet surfaces.

In our joint work [BS16] with Browning, Theorems 1.1 and 1.2 are used to study divisor sums of the shape

$$
\sum_{\substack{(s, t) \in \mathbb{Z}^{2} \\|s|,|t| \leqslant x}}\left(\prod_{i=1}^{n} \sum_{k_{i} \mid F_{i}(s, t)}\left(\frac{G_{i}(s, t)}{k_{i}}\right)\right),
$$

where $F_{i}, G_{i} \in \mathbb{Z}[s, t]$ are appropriate binary forms with $\sum_{i=1}^{n} \operatorname{deg}\left(F_{i}\right)=4$ and $(\vdots)$ denotes the Jacobi symbol. As a byproduct we provide matching upper and lower bounds agreeing with Manin's conjecture for every Châtelet surface and every quartic del Pezzo surface with a conic bundle structure over $\mathbb{Q}$. The example (1.6) can occur in the setting of quartic del Pezzo surfaces with a conic bundle structure. Indeed, let

$$
\Phi_{1}\left(x_{0}, \ldots, x_{4}\right):=x_{0} x_{1}-x_{2} x_{3}, \quad \Phi_{2}\left(x_{0}, \ldots, x_{4}\right):=x_{0}^{2}+2 x_{1}^{2}+x_{2}^{2}+x_{3}^{2}-x_{4}^{2}+x_{1} x_{3},
$$

and consider the surface $X \subset \mathbb{P}_{\mathbb{Q}}^{4}$ that is cut out by the system $\Phi_{1}=\Phi_{2}=0$. The map $f: X \rightarrow \mathbb{P}_{\mathbb{Q}}^{1}$ given by

$$
f(x):= \begin{cases}{\left[x_{0}, x_{2}\right]} & \text { if }\left(x_{0}, x_{2}\right) \neq(0,0) \\ {\left[x_{3}, x_{1}\right]} & \text { if }\left(x_{3}, x_{1}\right) \neq(0,0)\end{cases}
$$

is a conic bundle morphism whose fibers are given by

$$
\left(a^{2}+b^{2}\right) x^{2}+\left(a^{2}+a b+2 b^{2}\right) y^{2}=z^{2} .
$$

In the terminology of [BS16, §2] we have $\theta=\sqrt{-1}, K=\mathbb{Q}(\sqrt{-1}), G(s, t)=s^{2}+s t+2 t^{2}$, as well as $g(x)=x^{2}+x+2$ and $L=K(\sqrt{1+\sqrt{-1}})$. Furthermore, [BS16, Lemma 2.2] shows that

$$
h(s, t)=\sum_{k \mid s^{2}+t^{2}}\left(\frac{s^{2}+s t+2 t^{2}}{k}\right)
$$

can be written as a sum of the shape

$$
\sum_{\mathfrak{a} \mid(s-\sqrt{-1} t)} \psi_{K}(\mathfrak{a})
$$

where $\psi_{K}$ is a quadratic character in $\mathbb{Q}(\sqrt{-1})$ that coincides with the one in (1.6) because the character in (1.6) $)$ satisfies $\psi_{K}(\mathfrak{p})=1$ if and only if $\mathfrak{p}$ splits in $K(\sqrt{1+\sqrt{-1}})$.

Notation. The symbol $\mathfrak{p}$ will exclusively refer throughout this paper to prime ideals in $\mathscr{O}_{K}$ and the residue degree of any $\mathfrak{p} \subset \mathscr{O}_{K}$ will be denoted by $f_{\mathfrak{p}}$. We shall make frequent use of the multiplicative span of all linear prime ideals,

$$
\mathscr{P}_{K}^{\circ}=\left\{\mathfrak{a} \subset \mathscr{O}_{K}: \mathfrak{p} \mid \mathfrak{a} \Rightarrow f_{\mathfrak{p}}=1\right\}
$$


The symbols $\mu_{K}, \tau_{K}$ and $\Lambda_{K}$ will be used for the Möbius, divisor and the von Mangoldt function on $\mathscr{I}_{K}$, while $\omega_{K}$ will stand for the number of distinct prime ideal divisors on $\mathscr{I}_{K}$. Unless the contrary is explicitly stated, the implicit constants in Landau's $O$-notation and Vinogradov's «-notation are allowed to depend on $K$ and $\psi_{K}$ but no other parameters. Lastly, the notation $f(x) \asymp g(x)$ will be taken to mean $f(x) \ll g(x) \ll f(x)$.

Acknowledgement. We are grateful to Gérald Tenenbaum for his generous explanations. We are furthermore indebted to Régis de la Bretèche and Gérald Tenenbaum for providing us with the proof of Theorem 1.1.

\section{Precursory maneuvers}

We begin by establishing the following property,

$\mathfrak{a}, \mathfrak{b} \in \mathscr{I}_{K}$ coprime $\Rightarrow \Delta_{K}(\mathfrak{a} \mathfrak{b}) \leqslant \tau_{K}(\mathfrak{a}) \Delta_{K}(\mathfrak{b})$ and $\Delta_{K}\left(\mathfrak{a} \mathfrak{b} ; \psi_{K}\right) \leqslant \tau_{K}(\mathfrak{a}) \Delta_{K}\left(\mathfrak{b} ; \psi_{K}\right)$.

Indeed, any $\mathfrak{d} \mid \mathfrak{a} \mathfrak{b}$ can be written uniquely as $\mathfrak{d}=\mathfrak{d}_{1} \mathfrak{d}_{2}$, where $\mathfrak{d}_{1}\left|\mathfrak{a}, \mathfrak{d}_{2}\right| \mathfrak{b}$. Therefore

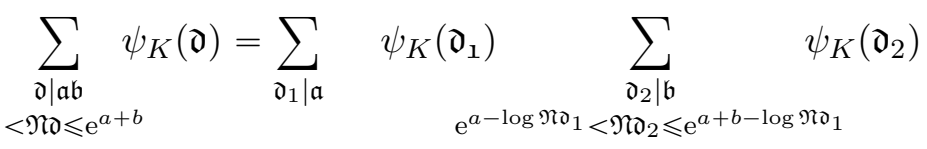

and a similar equality holds when $\psi_{K}$ is replaced by 1 . The triangle inequality ensures the validity of (2.1).

Lemma 2.1. For any $W_{0} \in \mathbb{N}$ and any $f: \mathscr{I}_{K} \rightarrow \mathbb{R}_{\geqslant 0}$ define the pair of functions

$$
M(x ; f):=1+\sup _{1 \leqslant y \leqslant x} \frac{1}{y} \sum_{\mathfrak{N a} \leqslant y} f(\mathfrak{a})
$$

and

$$
L\left(x, W_{0} ; f\right):=1+\sup _{1 \leqslant y \leqslant x} \frac{1}{\log y} \sum_{\substack{\mathfrak{N a} \leqslant y \\ \mathfrak{a} \in \mathscr{P}_{K}^{\circ} \\ g c d\left(\mathfrak{N a}, W_{0}\right)=1}} \frac{f(\mathfrak{a}) \mu_{K}(\mathfrak{a})^{2}}{\mathfrak{N a}} .
$$

If there exists $t>0$ such that $f(\mathfrak{a} \mathfrak{b}) \leqslant \tau_{K}(\mathfrak{a})^{t} f(\mathfrak{b})$ for all integral coprime ideals $\mathfrak{a}, \mathfrak{b}$ then for any $W_{0} \in \mathbb{N}$ we have the following as $x \rightarrow \infty$,

$$
M(x ; f) \asymp_{t, W_{0}} L\left(x, W_{0} ; f\right) .
$$

Proof. Let us begin by showing that

$$
\sum_{\mathfrak{N} \mathfrak{a} \leqslant x} \frac{f(\mathfrak{a})}{\mathfrak{N a}}=_{W_{0}} \sum_{\substack{\mathfrak{N} \mathfrak{a} \leqslant x \\ \mathfrak{a} \in \mathscr{P}_{K}^{\circ} \\ \operatorname{gcd}\left(\mathfrak{N a}, W_{0}\right)=1}} \frac{f(\mathfrak{a}) \mu_{K}(\mathfrak{a})^{2}}{\mathfrak{N a}} .
$$

The non-negativity of $f$ makes the inequality $\gg$ clear. To prove the remaining inequality we may factorise uniquely each $\mathfrak{a} \in \mathscr{I}_{K}$ as $\mathfrak{a}=\mathfrak{b} \mathfrak{c} \mathfrak{d}$, where each prime ideal divisor $\mathfrak{p}$ of $\mathfrak{b}$ satisfies $\mathfrak{N} \mathfrak{p} \mid W_{0}$ and each prime ideal factor of $\mathfrak{a}$ which is coprime to $W_{0}$ and has residue degree at least 2 divides $\mathfrak{c}$. The property of $f$ stated in our lemma shows that

$$
\sum_{\mathfrak{N} \mathfrak{a} \leqslant x} \frac{f(\mathfrak{a})}{\mathfrak{N a}} \leqslant \prod_{\mathfrak{N} \mathfrak{p} \mid W_{0}}\left(\sum_{m=0}^{\infty} \frac{\tau_{K}\left(\mathfrak{p}^{m}\right)^{t}}{\mathfrak{N} \mathfrak{p}^{m}}\right) \prod_{\substack{\mathfrak{N} \mathfrak{p} \leqslant x \\ f_{\mathfrak{p}} \neq 1}}\left(\sum_{m=0}^{\infty} \frac{\tau_{K}\left(\mathfrak{p}^{m}\right)^{t}}{\mathfrak{N} \mathfrak{p}^{m}}\right) \sum_{\substack{\mathfrak{N} \mathfrak{d} \leqslant x \\ \mathfrak{d} \in \mathscr{P}_{K}^{\circ} \\ \operatorname{gcd}\left(\mathfrak{N} \mathfrak{d}, W_{0}\right)=1}} \frac{f(\mathfrak{d})}{\mathfrak{N o}}
$$


and we see that the first term is $O_{t, W_{0}}(1)$. Writing $\mathfrak{N p}=p^{g}$ for a rational prime $p$ we see that the second product is

$$
\ll \prod_{2 \leqslant g \leqslant[K: \mathbb{Q}]} \prod_{p \leqslant x^{1 / g}}\left(\sum_{m=0}^{\infty} \frac{(m+1)^{t}}{p^{g m}}\right) \leqslant \prod_{p \leqslant x}\left(1+O_{t}\left(\frac{1}{p^{2}}\right)\right)^{[K: \mathbb{Q}]} \ll 1 .
$$

It thus remains to show that

$$
\sum_{\substack{\mathfrak{N} \mathfrak{d} \leqslant x \\ \mathfrak{d} \in \mathscr{P} \\ K}} \frac{f(\mathfrak{d})}{\mathfrak{N} \mathfrak{d}} \ll \sum_{\substack{\mathfrak{N} \mathfrak{d}_{1} \leq x \\ \mathfrak{d}_{1} \in \mathscr{P}_{K}^{\circ} \\ \operatorname{gcd}\left(\mathfrak{N} \mathfrak{d}_{1}, W_{0}\right)=1}} \frac{f\left(\mathfrak{d}_{1}\right) \mu_{K}\left(\mathfrak{d}_{1}\right)^{2}}{\mathfrak{N} \mathfrak{d}_{1}}
$$

To this end, we may factorise uniquely each $\mathfrak{d}$ as $\mathfrak{d}_{1} \mathfrak{d}_{2}$ where $\mathfrak{d}_{1}, \mathfrak{d}_{2}$ are coprime, $\mathfrak{d}_{1}$ is squarefree and $\mathfrak{d}_{2}$ is square-full. We may thus infer that

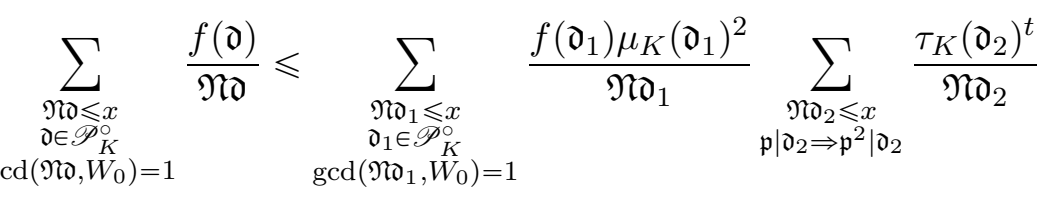

and the proof of $(2.2)$ is concluded by observing that the sum over $\mathfrak{d}_{2}$ is

$$
\leqslant \prod_{\mathfrak{N} \mathfrak{p} \leqslant x}\left(1+O_{t}\left(\frac{1}{\mathfrak{N p}^{2}}\right)\right)=O_{t}(1)
$$

In light of (2.2) it is sufficient for our lemma to show that

$$
M(x ; f) \asymp_{t} 1+\sup _{1 \leqslant y \leqslant x} \frac{1}{\log y} \sum_{\mathfrak{N a} \leqslant y} \frac{f(\mathfrak{a})}{\mathfrak{N a} .}
$$

Abel's summation can be employed to prove the inequality $\gg$ in (2.3). For the remaining inequality let us factorise $\mathfrak{a}$ as $\mathfrak{b} \mathfrak{c}$ with $\mathfrak{b}, \mathfrak{c}$ coprime, $\mathfrak{b}$ square-free and $\mathfrak{c}$ square-full. This yields

$$
\sum_{\mathfrak{N} \mathfrak{a} \leqslant y} f(\mathfrak{a}) \leqslant \sum_{\substack{\mathfrak{N} \mathfrak{c} \leqslant y \\ \mathfrak{p}\left|\mathfrak{c} \Rightarrow \mathfrak{p}^{2}\right| \mathfrak{c}}} \tau_{K}(\mathfrak{c})^{t} \sum_{\mathfrak{N} \mathfrak{b} \leqslant y / \mathfrak{N} \mathfrak{c}} f(\mathfrak{b}) \mu_{K}(\mathfrak{b})^{2}
$$

Therefore, if the following holds

$$
\sum_{\mathfrak{N} \mathfrak{b} \leqslant T} f(\mathfrak{b}) \mu_{K}(\mathfrak{b})^{2} \ll T^{\frac{1}{2}}+\frac{T}{\log T} \sum_{\mathfrak{N} \mathfrak{b} \leqslant T} \frac{f(\mathfrak{b})}{\mathfrak{N} \mathfrak{b}},
$$

then the required estimate (2.3) becomes available thanks to

$$
\begin{aligned}
& \sum_{\mathfrak{N} \mathfrak{a} \leqslant y} f(\mathfrak{a}) \ll y \sum_{\substack{\mathfrak{N} \mathfrak{c} \leqslant y \\
\mathfrak{p}\left|\mathfrak{c} \Rightarrow \mathfrak{p}^{2}\right| \mathfrak{c}}} \frac{\tau_{K}(\mathfrak{c})^{t}}{\mathfrak{N} \mathfrak{c}} \frac{1}{\log \frac{x}{\mathfrak{N} \mathfrak{c}}} \sum_{\mathfrak{N} \mathfrak{b} \leqslant x / \mathfrak{N} \mathfrak{c}} \frac{f(\mathfrak{b}) \mu_{K}(\mathfrak{b})^{2}}{\mathfrak{N b}} \\
& \ll y\left(\sup _{1 \leqslant y \leqslant x} \frac{1}{\log y} \sum_{\mathfrak{N} \mathfrak{b} \leqslant y} \frac{f(\mathfrak{b})}{\mathfrak{N \mathfrak { b }}}\right) \sum_{\substack{\mathfrak{N} \mathfrak{c} \leqslant y \\
\mathfrak{p}\left|\mathfrak{c} \Rightarrow \mathfrak{p}^{2}\right| \mathfrak{c}}} \frac{\tau_{K}(\mathfrak{c})^{t}}{\mathfrak{N} \mathfrak{c}}
\end{aligned}
$$


and

$$
\sum_{\substack{\mathfrak{N} \mathfrak{c} \leqslant y \\ \mathfrak{p}\left|\mathfrak{c} \Rightarrow \mathfrak{p}^{2}\right| \mathfrak{c}}} \frac{\tau_{K}(\mathfrak{c})^{t}}{\mathfrak{N} \mathfrak{c}} \ll \prod_{\mathfrak{N} \mathfrak{p} \leqslant y}\left(1+O_{t}\left(\frac{1}{\mathfrak{N p}^{2}}\right)\right) \ll_{t} 1
$$

To prove (2.4) we shall deploy the bound $f(\mathfrak{b}) \leqslant \tau_{K}(\mathfrak{b}) \ll \mathfrak{N b}^{1 / 2}$ to obtain

$$
\begin{aligned}
\sum_{\mathfrak{N} \mathfrak{b} \leqslant T} f(\mathfrak{b}) \mu_{K}(\mathfrak{b})^{2} & =\sum_{\mathfrak{N} \mathfrak{b} \leqslant T^{1 / 4}} f(\mathfrak{b}) \mu_{K}(\mathfrak{b})^{2}+\sum_{T^{1 / 4}<\mathfrak{N} \mathfrak{b} \leqslant T} f(\mathfrak{b}) \mu_{K}(\mathfrak{b})^{2} \\
& \ll T^{1 / 2}+\sum_{\mathfrak{N} \mathfrak{b} \leqslant T} f(\mathfrak{b}) \mu_{K}(\mathfrak{b})^{2} \frac{\log \mathfrak{N} \mathfrak{b}}{\log T} .
\end{aligned}
$$

Employing the identity $\mu_{K}(\mathfrak{b})^{2} \log \mathfrak{N \mathfrak { b }}=\sum_{\mathfrak{b}=\mathfrak{c}} \log \mathfrak{N p}$ allows us to bound the last sum by

$$
\frac{2}{\log T} \sum_{\mathfrak{N} \mathfrak{c} \leqslant T} f(\mathfrak{c}) \mu_{K}(\mathfrak{c})^{2} \sum_{\mathfrak{N} \mathfrak{p} \leqslant T / \mathfrak{N} \mathfrak{c}} \log \mathfrak{N} \mathfrak{p} \ll \frac{T}{\log T} \sum_{\mathfrak{N} \mathfrak{c} \leqslant T} \frac{f(\mathfrak{c})}{\mathfrak{N} \mathfrak{c}},
$$

where the prime number theorem for $K$ has been used.

Proposition 2.2. There exists a positive constant $c=c\left(K, \psi_{K}\right)$ such that for any $W \in \mathbb{N}$ we have

$$
\sum_{\substack{\mathfrak{N} \mathfrak{a} \leqslant x, \mathfrak{a} \in \mathscr{P}_{K}^{\circ} \\ \operatorname{gcd}(\mathfrak{N a}, W)=1}} \frac{\Delta_{K}\left(\mathfrak{a} ; \psi_{K}\right)^{2} \mu_{K}(\mathfrak{a})^{2}}{\mathfrak{N a}} \ll(\log x)^{1+c \hat{\varepsilon}(x)} .
$$

The implied constant is allowed to depend on $K, W$ and the character $\psi_{K}$.

Proof. The claim stems from Theorem 1.2 by taking $f(\mathfrak{a})=\Delta_{K}\left(\mathfrak{a} ; \psi_{K}\right)^{2}, t=2$ and $W_{0}=W$ in Lemma 2.1.

Lemma 2.1 makes possible to deduce Theorem 1.2 from the following claim: For any Dirichlet quadratic character $\psi_{K}$ there exist positive constants $c_{2}, z_{2}$ that depend only on $K$ and $\psi_{K}$ such that

$$
\sum_{\substack{\mathfrak{p} \mid \mathfrak{a} \Rightarrow \mathfrak{N} \mathfrak{p}>z_{2} \\ \mathfrak{a} \in \mathscr{P}}} \frac{\Delta\left(\mathfrak{a} ; \psi_{K}\right)^{2} \mu_{K}(\mathfrak{N})^{2}}{\mathfrak{N a} \leqslant x} \ll_{K, \psi_{K}}(\log x)^{1+c_{2} \widehat{\varepsilon}(x)}
$$

For $\mathfrak{a} \in \mathscr{I}_{K}, u \in \mathbb{R}$ and $q \in \mathbb{R}_{\geqslant 1}$ we let

$$
\Delta_{K}(\mathfrak{a} ; u):=\sum_{\substack{\mathfrak{d} \mid \mathfrak{a} \\ \mathrm{e}^{u}<\mathfrak{N} \mathfrak{d} \leqslant \mathrm{e}^{u+1}}} 1 \quad \text { and } \quad M_{q}(\mathfrak{a}):=\int_{-\infty}^{+\infty} \Delta_{K}(\mathfrak{a} ; u)^{q} \mathrm{~d} u .
$$

Lemma 2.3. For all $\mathfrak{a} \in \mathscr{I}_{K}$ and $q \in \mathbb{N}$ we have $M_{q}(\mathfrak{a}) \leqslant \tau_{K}(\mathfrak{a})^{q}$.

Proof. It is evident that $M_{q}(\mathfrak{a}) \leqslant \Delta_{K}(\mathfrak{a}) M_{q-1}(\mathfrak{a})$, hence the assertion can be validated by induction on $q$ upon noting that $M_{1}(\mathfrak{a})=\tau_{K}(\mathfrak{a})$.

Lemma 2.4. For each $\mathfrak{b} \in \mathscr{I}_{K}$ and positive integer a we have

$$
\sum_{\substack{\mathfrak{d}_{1}, \ldots, \mathfrak{d}_{a} \\ \mathfrak{d}_{i} \mid \mathfrak{b} \\ \max \mathfrak{N b}_{i}<\operatorname{emin} \mathfrak{N b}_{i}}} 1 \leqslant 2^{a+1} M_{a}(\mathfrak{b}) .
$$


Proof. It is convenient to rewrite the last summation condition as

$$
2-\log \left(\frac{\max \mathfrak{N b}_{i}}{\min \mathfrak{N b}_{i}}\right)>1
$$

hence, letting $x^{+}=\max \{0, x\}$ for $x \in \mathbb{R}$, we can bound the sum in the lemma by

$$
\sum_{\substack{\mathfrak{d}_{1}, \ldots, \mathfrak{d}_{a} \\ \mathfrak{d}_{i} \mid \mathfrak{b}}}\left(2-\log \left(\frac{\max \mathfrak{N b}_{i}}{\min \mathfrak{N b}_{i}}\right)\right)^{+}
$$

Using the convention $(a, b]=\varnothing$ when $a \geqslant b$ verifies the succeeding identity for all $a, b \in \mathbb{R}$,

$$
(b-a)^{+}=\int_{(a, b]} 1 \mathrm{~d} u .
$$

This provides the equality of the last sum with

$$
\sum_{\substack{\mathfrak{d}_{1}, \ldots, \mathfrak{d}_{a} \\ \mathfrak{d}_{i} \mid \mathfrak{b}}} \int_{\left[\log \max \mathfrak{N b}_{i}, 2+\log \min \mathfrak{N b}_{i}\right)} 1 \mathrm{~d} u=\int_{-\infty}^{+\infty}\left(\sum_{\substack{\mathrm{e}^{u-2}<\mathfrak{N} \mathfrak{d} \leqslant \mathrm{e}^{u} \\ \mathfrak{d} \mid \mathfrak{b}}} 1\right)^{a} \mathrm{~d} u
$$

which, upon decomposing the sum over $\mathfrak{d}$ as

$$
\sum_{\mathrm{e}^{u-2}<\mathfrak{N} \mathfrak{d} \leqslant \mathrm{e}^{u-1} \mathfrak{d} \mid \mathfrak{b}} 1+\sum_{\substack{\mathrm{e}^{u-1}<\mathfrak{N} \mathfrak{d} \leqslant \mathrm{e}^{u} \\ \mathfrak{d} \mid \mathfrak{b}}} 1
$$

leads to the desired bound

$$
2^{a} \int_{-\infty}^{+\infty}\left(\sum_{\substack{\mathrm{e}^{u-2}<\mathfrak{N} \mathfrak{d} \leqslant \mathrm{e}^{u-1} \\ \mathfrak{d} \mid \mathfrak{b}}} 1\right)^{a} \mathrm{~d} u+2^{a} \int_{-\infty}^{+\infty}\left(\sum_{\substack{\mathrm{e}^{u-1}<\mathfrak{N} \mathfrak{d} \leqslant \mathrm{e}^{u} \\ \mathfrak{d} \mid \mathfrak{b}}} 1\right)^{a} \mathrm{~d} u,
$$

that is clearly sufficient for the lemma.

For integers $c, q$ in the range $1 \leqslant c \leqslant q-1$ we can obtain via Hölder's inequality with exponents $\frac{q-1}{q-c}$ and $\frac{q-1}{c-1}$ the succeeding inequality

$$
M_{c}(\mathfrak{b})=\int_{-\infty}^{+\infty} \Delta(\mathfrak{b} ; u)^{\frac{q-c}{q-1}} \Delta(\mathfrak{b} ; u)^{\frac{q(c-1)}{q-1}} \mathrm{~d} u \leqslant M_{1}(\mathfrak{b})^{\frac{q-c}{q-1}} M_{q}(\mathfrak{b})^{\frac{c-1}{q-1}}
$$

Using this for $c=a$ and $c=q-a$ yields respectively

$$
M_{a}(\mathfrak{b}) \leqslant M_{1}(\mathfrak{b})^{\frac{q-a}{q-1}} M_{q}(\mathfrak{b})^{\frac{a-1}{q-1}} \text { and } M_{q-a}(\mathfrak{b}) \leqslant M_{1}(\mathfrak{b})^{\frac{a}{q-1}} M_{q}(\mathfrak{b})^{\frac{q-a-1}{q-1}}
$$

an inequality that will be used later.

\section{The proof of Theorem 1.2}

The bound (2.5) will be proved by an induction process which is given in $₫ 3.1$. The central result deployed in this process is Proposition 3.2, whose proof is postponed until 33.2 . 
3.1. The induction process. Throughout $\$ 3$ the positive real number $z_{2}=z_{2}\left(K, \psi_{K}\right)$ will be allowed to increase but it will be independent of the counting parameter $x$. The Erdös-Kac theorem for $K$ shows that the number of distinct prime ideal divisors of a typical element $\mathfrak{a} \in \mathscr{I}_{K}$ is of size $\log \log \mathfrak{N a}$, thus suggesting to consider the contribution of $\mathfrak{a}$ satisfying $\omega_{K}(\mathfrak{a})>10 \log \log x$ in (2.5). Using (2.1) with $\mathfrak{b}=\mathscr{O}_{K}$ we see that it is at most

$$
\sum_{\substack{\omega_{K}(\mathfrak{a})>10 \log \log x \\ \mathfrak{N a} \leqslant x}} \frac{\tau_{K}(\mathfrak{a})^{2} \mu_{K}(\mathfrak{a})^{2}}{\mathfrak{N a}} \leqslant \sum_{\mathfrak{N a} \leqslant x} \frac{\tau_{K}(\mathfrak{a})^{2} \mu_{K}(\mathfrak{a})^{2}}{\mathfrak{N a}}\left(\frac{5}{2}\right)^{\omega_{K}(\mathfrak{b})-10 \log \log x}
$$

and the inequality $10-10 \log \left(\frac{5}{2}\right)<1$ affirms the bound

$$
(\log x)^{-10 \log \left(\frac{5}{2}\right)} \prod_{\mathfrak{N} \mathfrak{p} \leqslant x}\left(1+\frac{10}{\mathfrak{N p}}\right) \ll \log x
$$

This shows that (2.5) stems from the estimate

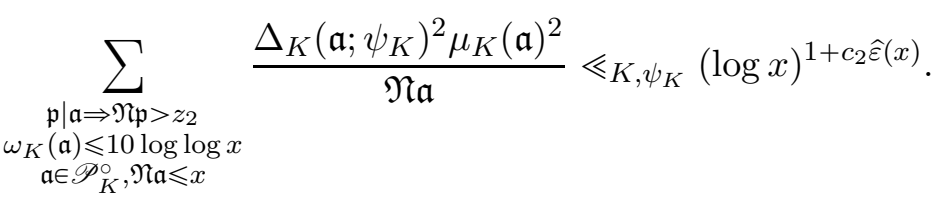

We will soon replace the $\Delta$-term by an expression involving an integral that approximates $\Delta_{K}\left(\mathfrak{a} ; \psi_{K}\right)$. The approximation can be performed when the divisors of $\mathfrak{a}$ are evenly spaced and we proceed by showing that the sum in (3.1) can be restricted to $\mathfrak{a}$ with this property. For any $A>0$ we define $\mathscr{E}(A)$ as the set of all $\mathfrak{a} \in \mathscr{I}_{K}$ for which there are distinct $\mathfrak{d}, \mathfrak{d}^{\prime}$ with

$$
\mathfrak{d}\left|\mathfrak{a}, \mathfrak{d}^{\prime}\right| \mathfrak{a}, \quad \mathfrak{N} \mathfrak{d} \leqslant \mathfrak{N o} \mathfrak{d}^{\prime} \leqslant \mathfrak{N o}\left(1+(\log 2 \mathfrak{N d})^{-A}\right) .
$$

Assume that $A \geqslant 10$. Then each ideal counted in

$$
\sum_{\substack{\mathfrak{p} \mid \mathfrak{a} \Rightarrow \mathfrak{T} \mathfrak{p}>z_{2} \\ \mathfrak{a} \in \mathscr{P}}} \frac{\Delta_{K}\left(\mathfrak{a} ; \psi_{K}\right)^{2} \mu_{K}(\mathfrak{a})^{2}}{\mathfrak{N a}(A), \mathfrak{N a} \leqslant x}
$$

is of the shape $\mathfrak{a}=\mathfrak{d} \mathfrak{d}^{\prime} \mathfrak{m}$, where $\mathfrak{d}, \mathfrak{d}^{\prime}, \mathfrak{m}$ are coprime in pairs and square-free and satisfy

$$
\mathfrak{N d} \leqslant \mathfrak{N d} \mathfrak{d}^{\prime} \leqslant \mathfrak{N o}\left(1+(\log 2 \mathfrak{N d})^{-A}\right) .
$$

Hence, by (2.1) with $\mathfrak{b}=\mathscr{O}_{K}$, the sum is bounded by

$$
\sum_{\substack{\mathfrak{m}, \mathfrak{d} \in \mathscr{P}>\\ \mathfrak{N m} \mathfrak{m} \mathfrak{K} \leqslant x}} \frac{\mu_{K}(\mathfrak{m})^{2} \mu_{K}(\mathfrak{d})^{2} \tau_{K}(\mathfrak{m})^{2}}{\mathfrak{N m} \mathfrak{N} \mathfrak{d} \tau_{K}(\mathfrak{m})^{-2}} \sum_{\substack{\mathfrak{d}^{\prime} \in \mathscr{P}_{K}^{\circ}, \mathfrak{p} \mid \mathfrak{a} \Rightarrow \mathfrak{N} \mathfrak{p}>z_{2} \\ \mathfrak{N} \mathfrak{d} \leqslant \mathfrak{N} \mathfrak{d}^{\prime} \leqslant \mathfrak{N} \mathfrak{d}\left(1+(\log 2 \mathfrak{N} \mathfrak{d})^{-A}\right)}} \frac{\mu_{K}\left(\mathfrak{d}^{\prime}\right)^{2} \tau_{K}\left(\mathfrak{d}^{\prime}\right)^{2}}{\mathfrak{N} \mathfrak{d}^{\prime}}
$$

Introducing the following arithmetic function,

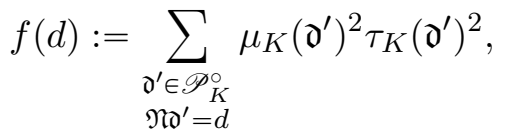

allows us to bound the sum over $\mathfrak{d}^{\prime}$ by

$$
\mathfrak{N o}^{-1} \sum_{\substack{p \mid d \Rightarrow p>z_{2} \\ \mathfrak{N} \leqslant \leqslant \leqslant \mathfrak{N}\left(1+(\log 2 \mathfrak{N} \mathfrak{d})^{-A}\right)}} f(d) .
$$


Using [Shi80, Th.1] shows that the last expression is bounded by $\mathfrak{N d}^{-1}(\log \mathfrak{N d})^{-1-A}$ multiplied by a quantity that is bounded by

$$
\ll_{A} \exp \left(\sum_{z_{2}<p \leqslant z_{2}+2 \mathfrak{N o}} f(p) / p\right) \ll \exp \left(\sum_{z_{2}<\mathfrak{N p} \leqslant z_{2}+2 \mathfrak{N o}} 4 / \mathfrak{N p}\right) \ll(\log \mathfrak{N d})^{4} .
$$

We have thus shown that the sum in (3.2) is

$$
\ll \sum_{\substack{\mathfrak{m} \in \mathscr{P}_{K}^{\circ} \\ \mathfrak{N} \mathfrak{m} \leqslant x}} \frac{\mu_{K}(\mathfrak{m})^{2} \tau_{K}(\mathfrak{m})^{2}}{\mathfrak{N m}} \sum_{\substack{\mathfrak{d} \in \mathscr{P}_{K}^{\circ} \\ \mathfrak{N} \mathfrak{d} \leqslant x}} \frac{\mu_{K}(\mathfrak{d})^{2} \tau_{K}(\mathfrak{d})^{2}}{\mathfrak{N} \mathfrak{d}(\log \mathfrak{N} \mathfrak{d})^{A-3}} \ll(\log x)^{4} \sum_{\substack{\mathfrak{d} \in \mathscr{P}_{K}^{\circ} \\ \mathfrak{N} \mathfrak{d} \leqslant x}} \frac{\mu_{K}(\mathfrak{d})^{2} \tau_{K}(\mathfrak{d})^{2}}{\mathfrak{N} \mathfrak{d}(\log \mathfrak{N} \mathfrak{d})^{A-3}}
$$

By Abel's summation the sum over $\mathfrak{d}$ is $\ll(\log x)^{7-A}$, thus yielding

$$
A \geqslant 10 \Rightarrow \sum_{\substack{\mathfrak{p} \mid \mathfrak{a} \Rightarrow \mathfrak{N} \mathfrak{p}>z_{2} \\ \mathfrak{a} \in \mathscr{P} \circ}} \frac{\tau_{K}(\mathfrak{a})^{2} \mu_{K}(\mathfrak{a})^{2}}{\mathfrak{N a}(A), \mathfrak{N a} \leqslant x}
$$

which reveals that, owing to (3.1), the next estimate is sufficient for the proof of (2.5),

$$
\sum_{\substack{\mathfrak{N} \mathfrak{a} \leqslant x, \mathfrak{p} \mid \mathfrak{a} \Rightarrow \mathfrak{N} \mathfrak{p}>z_{2} \\ \omega_{K}(\mathfrak{a} \leqslant 10 \log \log x \\ \mathfrak{a} \in \mathscr{P} \bigcirc}} \frac{\Delta_{K}\left(\mathfrak{a} ; \psi_{K}\right)^{2} \mu_{K}(\mathfrak{a})^{2}}{\mathfrak{N a}(10)} \ll_{K, \psi_{K}}(\log x)^{1+c_{2} \widehat{\varepsilon}(x)}
$$

The induction process that will enable us to prove (3.3) requires that we are in possession of an ordering of the prime ideals $\mathfrak{p} \subset \mathscr{O}_{K}$; thus we form the sequence $\left(\mathfrak{p}_{i}\right)_{i=1}^{\infty}$ such that

$$
i<j \Rightarrow \mathfrak{p}_{i} \neq \mathfrak{p}_{j}, \mathfrak{N} \mathfrak{p}_{i} \leqslant \mathfrak{N} \mathfrak{p}_{j}
$$

Prime ideals of the equal norm are allowed to be ordered arbitrarily, but their ordering is fixed once and for all. Hence, for any $\mathfrak{a}$ we can set $i^{+}(\mathfrak{a})=\max \left\{i \in \mathbb{N}: \mathfrak{p}_{i} \mid \mathfrak{a}\right\}$ and define

$$
\mathfrak{p}^{+}(\mathfrak{a}):=\mathfrak{p}_{i^{+}(\mathfrak{a})} .
$$

Furthermore, for each $r \in \mathbb{N}$ and square-free $\mathfrak{a} \in \mathscr{I}_{K}$, we let $\mathfrak{a}_{r}:=\mathfrak{a}$ if $r \geqslant \omega_{K}(\mathfrak{a})$. If $r<\omega_{K}(\mathfrak{a})$ holds then we choose the first $r$ prime ideal divisors of $\mathfrak{a}$ according to the ordering above and let $\mathfrak{a}_{r}$ be their product. Setting $r_{x}:=[10 \log \log x]$ shows that the sum in (3.3) is

$$
\sum_{\substack{\mathfrak{N} \mathfrak{a} \leqslant x, \mathfrak{p} \mid \mathfrak{a} \Rightarrow \mathfrak{N} \mathfrak{p}>z_{2} \\
\omega_{K}(\mathfrak{a}) \leqslant 10 \log \log x \\
\mathfrak{a} \in \mathscr{P}_{K}^{\circ}, \mathfrak{a} \notin \mathscr{E}(10)}} \frac{\Delta_{K}\left(\mathfrak{a}_{r_{x}} ; \psi_{K}\right)^{2} \mu_{K}(\mathfrak{a})^{2}}{\mathfrak{N a}} \leqslant \sum_{\substack { \mathfrak{N a} \leqslant x, \mathfrak{p} \mid \mathfrak{a} \Rightarrow \mathfrak{a} \mathfrak{a} \mathfrak{a}>z_{2} \\
\begin{subarray}{c}{K \\
\mathcal{P}_{K}{ \mathfrak { N a } \leqslant x , \mathfrak { p } | \mathfrak { a } \Rightarrow \mathfrak { a } \mathfrak { a } \mathfrak { a } > z _ { 2 } \\
\begin{subarray} { c } { K \\
\mathcal { P } _ { K } } }\end{subarray}} \frac{\Delta_{K}\left(\mathfrak{a}_{r_{x}} ; \psi_{K}\right)^{2} \mu_{K}(\mathfrak{a})^{2}}{\mathfrak{N a}} .
$$

Letting for any $\mathfrak{a} \in \mathscr{I}_{K}, a \in \mathbb{R}$ and $b \in(0,1]$,

$$
\Delta_{K}(\mathfrak{a} ; \psi ; a, b):=\left|\sum_{\substack{\mathrm{e}^{a}<\mathfrak{N} \mathfrak{d} \leqslant \mathrm{e}^{a+b} \mathfrak{d} \mid \mathfrak{a}\\}} \psi_{K}(\mathfrak{d})\right|
$$

sets the stage for the entrance of the important entity

$$
M_{q}\left(\mathfrak{a} ; \psi_{K}\right):=\int_{0}^{1} \int_{\mathbb{R}} \Delta_{K}(\mathfrak{a} ; \psi ; a, b)^{q} \mathrm{~d} a \mathrm{~d} b, \quad\left(\mathfrak{a} \in \mathscr{I}_{K}, q \in \mathbb{N}\right) .
$$


Let $q_{x}:=\left[\sqrt{r_{x} /\left(1+\log r_{x}\right)}\right]$ and define for $r, q \in \mathbb{N}$ the average

$$
\mathscr{L}(x):=4^{\frac{r_{x}}{q_{x}}} \log x+\sum_{\substack{\mathfrak{N} \mathfrak{a} \leqslant x, \mathfrak{a} \in \mathscr{P}_{K}^{\circ} \\ \mathfrak{p} \mid \mathfrak{a} \Rightarrow \mathfrak{N} \mathfrak{p}>z_{2}}} \frac{M_{2 q_{x}}\left(\mathfrak{a}_{r_{x}} ; \psi_{K}\right)^{\frac{1}{q_{x}}} \mu_{K}(\mathfrak{a})^{2}}{\mathfrak{N a}} .
$$

The next lemma shows that Theorem $[1.2$ stems from

$$
\mathscr{L}(x) \ll_{K, \psi_{K}}(\log x)^{1+c_{2} \hat{\varepsilon}(x)} .
$$

Lemma 3.1. For all $q \in \mathbb{N}$ and square-free $\mathfrak{a} \in \mathscr{I}_{K}$ with $\mathfrak{N a} \leqslant x$ and $\mathfrak{a} \notin \mathscr{E}(10)$ we have

$$
\Delta_{K}\left(\mathfrak{a} ; \psi_{K}\right)^{2} \leqslant 8^{2}+2^{10}(\log x)^{\frac{20}{q}} M_{2 q}\left(\mathfrak{a} ; \psi_{K}\right)^{\frac{1}{q}} .
$$

Proof. The lemma is valid if $\Delta_{K}\left(\mathfrak{a} ; \psi_{K}\right)<8$, we may therefore assume henceforth that the opposite holds. Note that the definition of $\Delta_{K}\left(\mathfrak{a} ; \psi_{K}\right)$ provides $a_{0} \in \mathbb{R}, b_{0} \in[0,1]$ such that

$$
\left|\Delta_{K}\left(\mathfrak{a} ; \psi_{K} ; a_{0}, b_{0}\right)\right| \geqslant \frac{1}{2} \Delta_{K}\left(\mathfrak{a} ; \psi_{K}\right) .
$$

We bring into play the box $\mathfrak{B} \subset \mathbb{R}^{2}$ given by

$$
\left(a_{0}, a_{0}+\frac{1}{8(\log 2 x)^{10}}\right) \times\left(b_{0}, b_{0}+\frac{1}{8(\log 2 x)^{10}}\right),\left(a_{0}-\frac{1}{8(\log 2 x)^{10}}, a_{0}\right) \times\left(b_{0}-\frac{1}{8(\log 2 x)^{10}}, b_{0}\right)
$$

respectively according to whether $b_{0}<\frac{1}{2}$ or not. We choose to focus on the latter case; the former being treated similarly. For any $(a, b) \in \mathfrak{B}$ we have

$$
\left|\Delta_{K}\left(\mathfrak{a} ; \psi_{K} ; a, b\right)-\Delta_{K}\left(\mathfrak{a}, \psi_{K} ; a_{0}, b_{0}\right)\right| \leqslant \sum_{\substack{\mathfrak{d} \mid \mathfrak{a} \\ \mathrm{e}^{a} \leqslant \mathfrak{N} \mathfrak{d} \leqslant \mathrm{e}^{a_{0}}}} 1+\sum_{\substack{\mathfrak{d} \mid \mathfrak{a} \\ \mathrm{e}^{a+b} \leqslant \mathfrak{N} \mathfrak{d} \leqslant \mathrm{e}^{a_{0}+b_{0}}}} 1 .
$$

If the first sum has more than one term then there exist $\mathfrak{d} \neq \mathfrak{d}^{\prime} \in \mathscr{I}_{K}$ with $\mathfrak{d}, \mathfrak{d}^{\prime} \mid \mathfrak{a}$ and

$$
\mathrm{e}^{a_{0}-1 / 8(\log 2 x)^{10}} \leqslant \mathfrak{N d} \leqslant \mathfrak{N o} \mathfrak{d}^{\prime} \leqslant \mathrm{e}^{u_{0}},
$$

thus leading via $\mathfrak{N a} \leqslant x$ and $\mathrm{e}^{z} \leqslant 1+2 z$ (valid in the range $0<z<1$ ), to

$$
\mathfrak{N d}^{\prime} \leqslant \mathfrak{N d} \mathrm{e}^{1 / 8(\log 2 x)^{10}} \leqslant \mathfrak{N o}\left(1+\frac{1}{4(\log 2 x)^{10}}\right) \leqslant \mathfrak{N o}\left(1+\frac{1}{(\log 2 \mathfrak{N} \mathfrak{d})^{10}}\right)
$$

which contradicts the assumption $\mathfrak{a} \notin \mathscr{E}(10)$ of our lemma. A similar argument shows that the second sum in (3.8) also contains at most one term, therefore invoking $\Delta_{K}\left(\mathfrak{a} ; \psi_{K}\right) \geqslant 8$ and (3.7) provides us with

$$
\Delta_{K}\left(\mathfrak{a} ; \psi_{K} ; a, b\right) \geqslant \frac{\Delta_{K}\left(\mathfrak{a} ; \psi_{K}\right)}{2}-2 \geqslant \frac{\Delta_{K}\left(\mathfrak{a} ; \psi_{K}\right)}{4} .
$$

This inequality immediately furnishes the required estimate by restricting the range of integration in (3.5) to $\mathfrak{B}$.

For positive integers $r, q$ and any $\sigma \in\left(0, \frac{1}{4}\right]$ we define the functions

$$
L_{r, q}^{*}(\sigma):=\frac{4^{\frac{r}{q}}}{\sigma}+\sum_{\substack{\mathfrak{a} \in \mathscr{P}_{K}^{\circ} \\ \mathfrak{p} \mid \mathfrak{a} \Rightarrow \mathfrak{N} \mathfrak{p}>z_{2}}} \frac{M_{2 q}\left(\mathfrak{a}_{r} ; \psi_{K}\right)^{\frac{1}{q}} \mu_{K}(\mathfrak{a})^{2}}{\mathfrak{N a}^{1+\sigma}}
$$


and for $s \in \mathbb{R}_{\geqslant 1}$ we let

$$
f(s):=\sqrt{\frac{s}{1+\log s}} .
$$

Noting that $\mathscr{L}(x) \leqslant \mathrm{e} L_{r_{x}, q_{x}}^{*}(1 / \log x)$, our aim now becomes to prove that for all sufficiently small $\sigma>0$ we have

$$
r \gg 1, q=[f(r)] \Rightarrow L_{r, q}^{*}(\sigma) \ll \frac{\mathrm{e}^{c_{2} \sqrt{r \log r}}}{\sigma}
$$

for some constant $c_{2}>0$ depending at most on $K$ and $\psi_{K}$. Clearly, this is sufficient for verifying (3.6).

The strategy for the proof of (3.9) is indirect and resembles a backwards induction process. First, note that if the variable $r$ is replaced by any fixed integer constant $t$, then for any $q$ we have $L_{t^{\prime}, q}^{*} \ll_{t^{\prime}} 1 / \sigma$. Indeed, using Lemma 2.3 in combination with the obvious bound $M_{2 q}\left(\mathfrak{a} ; \psi_{K}\right) \leqslant M_{2 q}(\mathfrak{a})$ furnishes

$$
L_{t^{\prime}, q}^{*}(\sigma)-\frac{4^{\frac{t^{\prime}}{q}}}{\sigma} \ll \sum_{\mathfrak{a} \in \mathscr{I}_{K}} \frac{\tau_{K}\left(\mathfrak{a}_{t^{\prime}}\right)^{2} \mu_{K}(\mathfrak{a})^{2}}{\mathfrak{N} \mathfrak{a}^{1+\sigma}} \leqslant \sum_{\mathfrak{a} \in \mathscr{I}_{K}} \frac{2^{2 t^{\prime}}}{\mathfrak{N a}^{1+\sigma}} \ll_{t^{\prime}} \zeta_{K}(1+\sigma) \ll_{t}^{\prime} \frac{1}{\sigma} .
$$

It will therefore be advantageous to bound $L_{r, q}^{*}(\sigma)$ in terms of $L_{r-1, q}^{*}(\sigma)$ for $r$ and $q$ in suitable ranges. To this end we shall deploy the succeeding lemma, whose proof is postponed until §.2.

Proposition 3.2. There exist positive constants $c_{3}, t^{\prime}, z_{2}, \sigma_{0}$ that depend at most on $K$ and $\psi_{K}$, such that for all integers $t, m$ in the range

$$
t^{\prime} \leqslant t \leqslant 10 \log \frac{1}{\sigma}, \quad m \leqslant \sqrt{t /(1+\log t)}<m+2,
$$

and $\sigma \in\left(0, \sigma_{0}\right)$ we have

$$
L_{t, m}^{*}(\sigma) \leqslant \mathrm{e}^{\frac{c_{3}}{m}} L_{t-1, m}^{*}(\sigma) .
$$

To deduct (3.6) from Proposition 3.2 define for each integer $\ell$ the following set,

$$
\mathscr{A}_{\ell}:=\{n \in \mathbb{N}: \ell \leqslant f(n)<\ell+1\},
$$

which furnishes the following partition into disjoint sets

$$
\mathbb{N} \cap\left[t^{\prime}, r\right]=\bigcup_{\ell \in \mathbb{N}} \mathscr{A}_{\ell}
$$

Let $k:=\min \mathscr{A}_{\ell}$. It is easy to see that $f(k+c \sqrt{k \log k})>f(k)+1$ holds for some large positive $c$ independent of $k$, and therefore $\sharp \mathscr{A}_{\ell} \leqslant c \sqrt{\ell \log \ell}$. In addition, the definition of $k$ shows that $f(k-1)<\ell$ and therefore $\sqrt{k \log k} \ll \ell \log \ell$, hence $\sharp \mathscr{A}_{\ell} \leqslant c_{4} q \log \ell$ for some absolute constant $c_{4}>0$. Furthermore, $\mathscr{A}_{\ell}$ will be empty unless $\ell \leqslant q$.

It is now time to reveal our backwards induction process. Whenever $n \in \mathscr{A}_{q}$ we use Proposition 3.2 with $t=n, m=q$ to reduce the value of $n$ from $r$ down to min $\mathscr{A}_{q}$. This will come at a cost of $\exp \left(\frac{c_{3}}{q} \sharp \mathscr{A}_{q}\right) \leqslant q^{c_{3} c_{4}}$. At the end of this section we shall prove that there exists a positive constant $c_{5}=c_{5}\left(K, \psi_{K}\right)$ such that for all $\sigma>0$ sufficiently small we have

$$
t^{\prime} \leqslant t \leqslant 10 \log \frac{1}{\sigma}, m=[f(t)] \Rightarrow L_{t, m}^{*}(\sigma) \leqslant m^{c_{5}} L_{t, m-1}^{*}(\sigma) .
$$

When $n$ reaches $\min \mathscr{A}_{q}$ we will use (3.11) with $t=\min \mathscr{A}_{q_{x}}$ and $m=q$. We subsequently iterate the process by using Proposition 3.2 with $m=q-1$ and $t=n$ for all $n \in \mathscr{A}_{q-1}$. We 
repeat this procedure going backwards until $\ell$ is small enough so that $t^{\prime} \in \mathscr{A}_{\ell}$. The total cost will be

$$
\ll \prod_{\ell \leqslant q} \ell^{c_{3} c_{4}+c_{5}} \leqslant \mathrm{e}^{c_{2} \sqrt{r \log r}},
$$

for some constant $c_{2}>0$ that depends at most on $K$ and $\psi_{K}$. At the end of this process we shall be left with $L_{t^{\prime}, q}^{*}(\sigma)$ which can be estimated via (3.10), thus concluding the proof of (3.9).

Proof of (3.11). Let us begin by introducing the constants $\eta_{K}:=\min \left\{10^{-3}, 2^{-\frac{3}{[K: \mathbb{Q}}}\right\}$ and $u_{t}:=\exp \left(70^{t} / t\right)$. We shall make use of the set $\mathscr{D}_{t}$ that consists of all square-free $\mathfrak{a} \in \mathscr{I}_{K}$ for which there are distinct $\mathfrak{d}, \mathfrak{d}^{\prime}$ satisfying

$$
\mathfrak{d}\left|\mathfrak{a}_{t}, \mathfrak{d}^{\prime}\right| \mathfrak{a}_{t}, \quad \mathfrak{N o} \leqslant \mathfrak{N o} \mathfrak{d}^{\prime} \leqslant \mathfrak{N o}\left(1+\eta_{K}^{t}\right) .
$$

For each such $\mathfrak{a}$ we can choose and fix square-free and coprime in pairs $\mathfrak{d}_{\mathfrak{a}_{t}}, \mathfrak{d}_{\mathfrak{a}_{t}}^{\prime}, \mathfrak{m}_{\mathfrak{a}_{t}} \in \mathscr{I}_{K}$ with $\mathfrak{a}=\mathfrak{d}_{\mathfrak{a}_{t}} \mathfrak{d}_{\mathfrak{a}_{t}}^{\prime} \mathfrak{m}_{\mathfrak{a}_{t}}$ and $\mathfrak{d}_{\mathfrak{a}_{t}}, \mathfrak{d}_{\mathfrak{a}_{t}}^{\prime}$ being in the range designated above. We may now deploy the inequality $\mu_{K}(\mathfrak{a})^{2} M_{2 m}\left(\mathfrak{a}_{t} ; \psi_{K}\right) \leqslant \mu_{K}(\mathfrak{a})^{2} \tau_{K}\left(\mathfrak{a}_{t}\right)^{2 m}=4^{t m}$ to infer that for large enough $t \geqslant t^{\prime}$ the contribution of $\mathfrak{a}$ with $\mathfrak{N o}_{\mathfrak{a}_{t}} \leqslant \exp \left(70^{t}\right)$ towards $L_{t, m}^{*}(\sigma)-4^{\frac{t}{m}} / \sigma$ is at most

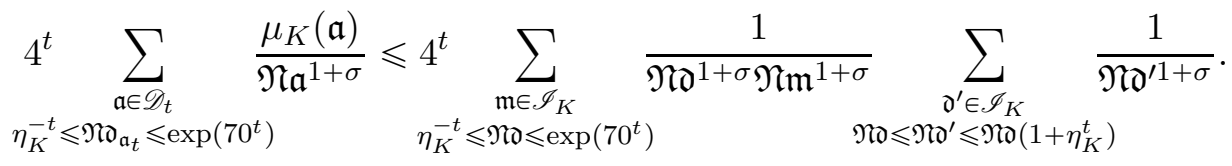

The estimate $\sum_{\mathfrak{N o} \mathfrak{d}^{\prime} \leqslant x} 1=c_{K} x+O_{K}\left(x^{1-\frac{1}{[K: \mathbb{Q}]}}\right)$ shows that the sum over $\mathfrak{d}^{\prime}$ is

$$
\leqslant \mathfrak{N o}^{-1-\sigma}\left(c_{K} \eta_{K}^{t} \mathfrak{N o}+O_{K}\left(\mathfrak{N d}{ }^{1-\frac{1}{[K: \mathbb{Q}]}}\right)\right)
$$

which provides the following bound,

$$
\ll \frac{4^{t}}{\sigma}\left(\eta_{K}^{t} \sum_{\mathfrak{N} \mathfrak{d} \leqslant \exp \left(70^{t}\right)} \frac{1}{\mathfrak{N} \mathfrak{d}}+\sum_{\mathfrak{N} \mathfrak{d} \geqslant \eta_{K}^{-t}} \frac{1}{\mathfrak{N D}^{1+[K: \mathbb{Q}]}}\right) \ll \frac{4^{t}}{\sigma}\left(\eta_{K}^{t} 70^{t}+\eta_{K}^{t[K: \mathbb{Q}]}\right) \ll \frac{1}{\sigma 2^{t}} .
$$

Let us now focus on the contribution of $\mathfrak{a} \in \mathscr{D}_{t}$ with $\mathfrak{N o}_{\mathfrak{a}_{t}}>\exp \left(70^{t}\right)$. The cardinality of the prime ideal divisors of $\mathfrak{a}$ in the range $\mathfrak{N} \mathfrak{p} \leqslant \exp \left(70^{t} / t\right)$, henceforth denoted by $\omega(\mathfrak{a} ; t)$, cannot exceed $t$, otherwise the first $t$ prime ideals dividing $\mathfrak{a}$ will have norm in that range, thus $\mathfrak{N o}_{\mathfrak{a}_{t}} \leqslant \mathfrak{N a}_{t} \leqslant\left(\exp \left(70^{t} / t\right)\right)^{t}$, which is contradiction. In the case where $\sigma>(32 / 9) t 70^{-t}$ we see, upon using $\zeta_{K}(1+\sigma) \ll \sigma^{-1}$, that the contribution of the ideals $\mathfrak{a}$ under consideration towards $L_{t, m}^{*}(\sigma)-4^{\frac{t}{m}} / \sigma$ is at most

$$
4^{t} \sum_{\mathfrak{a} \subset \mathscr{O}_{K}} \frac{\mu_{K}(\mathfrak{a})^{2} \mathfrak{N a a _ { t } ^ { \sigma / 2 }}}{\mathfrak{N a}^{1+\sigma} u_{t}^{t \sigma / 2}} \ll 4^{t} \sum_{\mathfrak{a} \subset \mathscr{O}_{K}} \frac{\mu_{K}(\mathfrak{a})^{2}}{\mathfrak{N a}^{1+\sigma / 2} u_{t}^{t \sigma / 2}} \ll \frac{(7 / 10)^{t}}{\sigma} .
$$

In the remaining case $\sigma \leqslant(32 / 9) t 70^{-t}$ we set $v:=2 /(\log 70)$ and bound the contribution by

$$
4^{t} \sum_{\mathfrak{a} \subset \mathscr{O}_{K}} \frac{\mu_{K}(\mathfrak{a})^{2} v^{\omega(\mathfrak{a} ; t)-t}}{\mathfrak{N a} \mathfrak{a}^{1+\sigma}} \ll \frac{4^{t}}{v^{t} \sigma} \prod_{\mathfrak{N} \mathfrak{p} \leqslant \exp \left\{\sqrt{u_{t}}\right\}}\left(1+\mathfrak{N p}^{-1}\right)^{v-1}
$$


which is again $\ll(7 / 10)^{t} / \sigma$. Thus far we have shown that

$$
L_{t, m}^{*}(\sigma) \ll \frac{4^{\frac{t}{m}}+(7 / 10)^{t}}{\sigma}+\sum_{\substack{\mathfrak{a} \in \mathscr{P}_{K}^{\circ}, \mathfrak{a} \notin \mathscr{D}_{t} \\ \mathfrak{p} \mid \mathfrak{a} \Rightarrow \mathfrak{N} \mathfrak{p}>z_{2}}} \frac{M_{2 m}\left(\mathfrak{a}_{t} ; \psi_{K}\right)^{\frac{1}{m}} \mu_{K}(\mathfrak{a})^{2}}{\mathfrak{N a}^{1+\sigma}} .
$$

Taking $z_{2}>2$ we see that each $\mathfrak{a}$ in the sum has odd norm, thus each element of the set

$$
S:=\{(a, b) \in(-1,0] \times(0,1): a+b \geqslant 0\}
$$

satisfies $\mathbb{Z} \cap\left(\mathrm{e}^{u}, \mathrm{e}^{a+b}\right]=\{1\}$ and therefore $\Delta_{K}\left(\mathfrak{a} ; \psi_{K} ; a, b\right)=1$. Hence, for any $q \in \mathbb{N}$ we have $M_{2 q}\left(\mathfrak{a} ; \psi_{K}\right) \geqslant \operatorname{vol}(S)=1 / 2$. We can now imitate the proof of Lemma 3.1, replacing $1 /(\log 2 x)^{10}$ by $\eta_{K}^{t}$, to prove that for all $q \in \mathbb{N}$ and square-free $\mathfrak{a} \in \mathscr{I}_{K}$ with $\mathfrak{a} \notin \mathscr{D}_{t}$ we have

$$
\Delta_{K}\left(\mathfrak{a}_{t} ; \psi_{K}\right)^{2} \leqslant 8^{2}+2^{10} \eta_{K}^{-\frac{2 t}{q}} M_{2 q}\left(\mathfrak{a} ; \psi_{K}\right)^{\frac{1}{q}} \leqslant 2^{11} \eta_{K}^{-\frac{2 t}{q}} M_{2 q}\left(\mathfrak{a}_{t} ; \psi_{K}\right)^{\frac{1}{q}} .
$$

Using this for $q=m-1$ in combination with

$$
M_{2 m}\left(\mathfrak{a}_{t} ; \psi_{K}\right) \leqslant \Delta_{K}\left(\mathfrak{a}_{t} ; \psi_{K}\right)^{2} M_{2 m-2}\left(\mathfrak{a}_{t} ; \psi_{K}\right)
$$

leads to

$$
M_{2 m}\left(\mathfrak{a}_{t} ; \psi_{K}\right)^{\frac{1}{m}} \leqslant 2^{\frac{11}{m}} \eta_{K}^{-\frac{2 t}{m(m-1)}} M_{2 m-2}\left(\mathfrak{a}_{t} ; \psi_{K}\right)^{\frac{1}{m-1}} .
$$

The proof of (3.11) is concluded by injecting the last inequality into (3.12) and making use of $m \gg f(t)$ to derive $\eta_{K}^{-\frac{2 t}{m(m-1)}} \leqslant m^{c_{5}}$ for some positive constant $c_{5}$ that depends at most on $K$ and $\psi_{K}$.

3.2. The proof of Proposition 3.2. To relate $L_{t, m}^{*}(\sigma)$ and $L_{t-1, m}^{*}(\sigma)$ demands that we have an understanding of the fluctuation of $M_{2 m}\left(\mathfrak{a} ; \psi_{K}\right)^{\frac{1}{m}}$ as the number of prime ideal divisors of $\mathfrak{a}$ varies. To this end, we observe that for any $\mathfrak{a} \in \mathscr{I}_{K}$ and prime $\mathfrak{p}$ we have

$$
\Delta_{K}\left(\mathfrak{a} ; \psi_{K} ; a, b\right)=\Delta_{K}\left(\mathfrak{a} ; \psi_{K} ; a, b\right)+\psi_{K}(\mathfrak{p}) \Delta_{K}\left(\mathfrak{a}, \psi_{K} ; a-\log \mathfrak{N p}, b\right) .
$$

For a positive integer $m$ we can raise to the power $2 m$ to obtain

$$
\Delta_{K}\left(\mathfrak{a} \mathfrak{p} ; \psi_{K} ; a, b\right)^{2 m}=\sum_{0 \leqslant j \leqslant 2 m}\left(\begin{array}{c}
2 m \\
j
\end{array}\right) \psi(\mathfrak{p})_{K}^{2 m-j} \Delta_{K}\left(\mathfrak{a} ; \psi_{K} ; a, b\right)^{j} \Delta_{K}\left(\mathfrak{a} \mathfrak{p} ; \psi_{K} ; a-\log \mathfrak{N \mathfrak { p }}, b\right)^{q-j}
$$

Hence, letting for $\mathfrak{a} \in \mathscr{I}_{K}, w \in \mathbb{R}, m \in \mathbb{N}$ and $0 \leqslant j \leqslant m$,

$$
N_{j, m}(\mathfrak{a}, w):=\int_{0}^{1} \int_{\mathbb{R}} \Delta_{K}\left(\mathfrak{a} ; \psi_{K} ; a, b\right)^{j} \Delta_{W}(\mathfrak{a}, \psi ; a-w, b)^{q-j} \mathrm{~d} a \mathrm{~d} b
$$

and recalling (3.5) we arrive at

$$
M_{2 m}\left(\mathfrak{a} \mathfrak{p} ; \psi_{K}\right)=2 M_{2 m}\left(\mathfrak{a} ; \psi_{K}\right)+\sum_{1 \leqslant j \leqslant 2 m-1}\left(\begin{array}{c}
2 m \\
j
\end{array}\right) \psi_{K}(\mathfrak{p})^{j} N_{j, 2 m}(\mathfrak{a}, \log \mathfrak{N} \mathfrak{p}) .
$$

If $1<j<m-1$ we use $c d \leqslant \frac{c^{2}}{2}+\frac{d^{2}}{2}$ for

$$
c=\Delta_{K}\left(\mathfrak{a} ; \psi_{K} ; a, b\right)^{j+1} \Delta_{K}\left(\mathfrak{a} ; \psi_{K} ; a-w, b\right)^{m-j-1}, d=\Delta_{K}(\mathfrak{a} ; \psi ; a, b)^{j} \Delta_{K}\left(\mathfrak{a} ; \psi_{K} ; a-w, b\right)^{m-j}
$$

to acquire

$$
N_{2 j+1,2 m}(\mathfrak{a}, w) \leqslant \frac{1}{2} N_{2 j+2,2 m}(\mathfrak{a}, w)+\frac{1}{2} N_{2 j, 2 q}(\mathfrak{a}, w)
$$


and the inequality $c d \leqslant \frac{m c^{2}}{2}+\frac{d^{2}}{2 m}$ yields in like manner

$$
\begin{aligned}
N_{1,2 m}(\mathfrak{a}, w) & \leqslant \frac{m}{2} N_{2,2 m}(\mathfrak{a}, w)+\frac{1}{2 m} M_{2 m}\left(\mathfrak{a} ; \psi_{K}\right), \\
N_{2 m-1,2 m}(\mathfrak{a}, w) & \leqslant \frac{m}{2} N_{2 m-2,2 m}(\mathfrak{a}, w)+\frac{1}{2 m} M_{2 m}\left(\mathfrak{a} ; \psi_{K}\right) .
\end{aligned}
$$

Putting everything together, we have

$$
M_{2 m}\left(\mathfrak{a} \mathfrak{p} ; \psi_{K}\right) \leqslant 4 M_{2 m}\left(\mathfrak{a} ; \psi_{K}\right)+W_{2 m}(\mathfrak{a}, \mathfrak{p})
$$

where

$$
W_{2 m}(\mathfrak{a}, \mathfrak{p}):=\sum_{1 \leqslant j \leqslant m-1} b_{j}\left(\begin{array}{c}
2 m \\
2 j
\end{array}\right) N_{2 j, 2 m}(\mathfrak{a}, \log \mathfrak{N} \mathfrak{p})
$$

and the sequence given through

$$
b_{j}:= \begin{cases}1+\frac{m}{2 m-1}+\frac{2}{3}(m-2) & \text { if } j=1 \\ 1+\frac{m}{2 m-1} & \text { if } j=m-1 \\ 1+\frac{j}{2 m-2 j-1}+\frac{m-j}{2 j+1} & \text { otherwise. }\end{cases}
$$

satisfies $b_{j} \leqslant 1+\frac{2}{3} m$.

Assume that we are given $\mathfrak{a} \in \mathscr{I}_{K}$ with $\omega_{K}(\mathfrak{a})>t-1$. Then letting $\mathfrak{p}_{t}(\mathfrak{a})$ be the $t$-th prime ideal factor of $\mathfrak{a}$ according to the ordering (3.4) and using $\left(y_{1}+y_{2}\right)^{\frac{1}{m}} \leqslant y_{1}^{\frac{1}{m}}+y_{2}^{\frac{1}{m}}$, valid for $y_{i} \in \mathbb{R}_{\geqslant 0}$, we deduce

$$
M_{2 m}\left(\mathfrak{a}_{t} ; \psi_{K}\right)^{\frac{1}{m}} \leqslant 4^{\frac{1}{m}} M_{2 m}\left(\mathfrak{a}_{t-1} ; \psi_{K}\right)^{\frac{1}{m}}+W_{2 m}\left(\mathfrak{a}_{t-1}, \mathfrak{p}_{t}(\mathfrak{a})\right)^{\frac{1}{m}} .
$$

This inequality is also valid if $\omega_{K}(\mathfrak{a}) \leqslant t-1$, since in that case we have $\mathfrak{a}_{t}=\mathfrak{a}_{t-1}$. We obtain

$$
L_{t, m}^{*}(\sigma) \leqslant 4^{\frac{1}{m}} L_{t-1, m}^{*}(\sigma)+\sum_{\substack{\mathfrak{m} \in \mathscr{P}_{K}^{\circ} \\ \omega_{K}(\mathfrak{m})=t-1 \\ \mathfrak{p} \mid \mathfrak{m} \Rightarrow \mathfrak{N} \mathfrak{p}>z_{2}}} \sum_{\substack{\mathfrak{p}_{j} \in_{K}^{\circ} \\ j>i^{+}(\mathfrak{m})}} W_{2 m}\left(\mathfrak{m}, \mathfrak{p}_{j}\right)^{\frac{1}{m}} \sum_{\mathfrak{n}_{t}=\mathfrak{m p}_{j}} \frac{\mu_{K}^{2}(\mathfrak{n})}{\mathfrak{N n}^{1+\sigma}}
$$

Each ideal $\mathfrak{n}$ is of the form $\mathfrak{m}_{j} \mathfrak{d}$, where $\mathfrak{d}$ is square-free and each prime divisor of $\mathfrak{d}, \mathfrak{p}_{i} \mid \mathfrak{d}$ satisfies $i>j$. We can therefore deduce that the sum over $\mathfrak{n}$ is

$$
\ll \sum_{\mathfrak{n}_{t}=\mathfrak{m} \mathfrak{p}_{j}} \frac{\mu_{K}^{2}(\mathfrak{n})}{\mathfrak{N \mathfrak { n }}^{1+\sigma}} \ll \mathfrak{N m}_{\mathfrak{m} \mathfrak{p}_{j}^{-1-\sigma}} \prod_{\mathfrak{N} \mathfrak{p}>\mathfrak{N \mathfrak { p }}_{j}}\left(1+\frac{1}{\mathfrak{N p}^{1+\sigma}}\right)
$$

and, recalling that we denote the Dedekind zeta function of $K$ by $\zeta_{K}$, we deduce that the last product is

$$
\leqslant \zeta_{K}(1+\sigma) \prod_{\mathfrak{N} \mathfrak{p} \leqslant \mathfrak{N} \mathfrak{p}_{j}}\left(1+\frac{1}{\mathfrak{N p}^{1+\sigma}}\right)^{-1} \ll \frac{1}{\sigma} \prod_{\mathfrak{N} \mathfrak{p} \leqslant \mathfrak{N p}_{j}}\left(1-\frac{1}{\mathfrak{N p}^{1+\sigma}}\right) .
$$

The inequality $\mathfrak{N p}^{-\sigma} \geqslant 1-\sigma \log \mathfrak{N p}$ and Mertens' theorem show that the inner product is

$$
\ll \prod_{\mathfrak{N} \mathfrak{p} \leqslant \mathfrak{N} \mathfrak{p}_{j}}\left(1-\frac{1}{\mathfrak{N p}}\right) \exp \left(\sigma \sum_{\mathfrak{N} \mathfrak{p} \leqslant \mathfrak{N} \mathfrak{p}_{j}} \frac{\log \mathfrak{N p}}{\mathfrak{N p}}\right) \ll \frac{\mathfrak{N p}_{j}^{\sigma}}{\log \mathfrak{N p}_{j}}
$$


thus showing that the sum over $\mathfrak{n}$ is $\ll_{K} \sigma^{-1} \mathfrak{N m}^{-1-\sigma}\left(\mathfrak{N p}_{j} \log \mathfrak{N p}_{j}\right)^{-1}$. Letting for $\mathfrak{m} \in \mathscr{P}_{K}^{\circ}$,

$$
\mathscr{A}_{m}(\mathfrak{m}):=\sum_{\substack{\mathfrak{p}_{j} \in \mathscr{P}_{K}^{\circ} \\ j>i^{+}(\mathfrak{m})}} \frac{W_{2 m}\left(\mathfrak{m}, \mathfrak{p}_{j}\right)^{\frac{1}{m}}}{\mathfrak{N p}_{j} \log \mathfrak{N p}_{j}}
$$

we have thus obtained

$$
L_{t, m}^{*}(\sigma)-4^{\frac{1}{m}} L_{t-1, m}^{*}(\sigma) \ll \frac{1}{\sigma} \sum_{\substack{\mathfrak{m} \in \mathscr{P} \supset_{K}^{\circ} \\ \omega_{K}(\mathfrak{m})=t-1 \\ \mathfrak{p} \mid \mathfrak{m} \Rightarrow \mathfrak{T} \mathfrak{p}>z_{2}}} \frac{\mu_{K}^{2}(\mathfrak{m})}{\mathfrak{N m} \mathfrak{m}^{1+\sigma}} \mathscr{A}_{m}(\mathfrak{m}) .
$$

Using Hölder's inequality with exponents $m, \frac{m}{m-1}$ we see that $\mathscr{A}_{m}(\mathfrak{m})$ is at most

$$
\left(\sum_{\substack{\mathfrak{p}_{j} \in \mathscr{P}_{K}^{\circ} \\ j>i^{+}(\mathfrak{m})}} \frac{W_{2 m}\left(\mathfrak{m}, \mathfrak{p}_{j}\right) \log \mathfrak{N} \mathfrak{p}_{j}}{\mathfrak{N p}_{j}}\right)^{\frac{1}{m}}\left(\sum_{\substack{\mathfrak{p}_{j} \in \mathscr{P}_{K}^{\circ} \\ j>i^{+}(\mathfrak{m})}} \frac{1}{\mathfrak{N p}_{j}\left(\log \mathfrak{N} \mathfrak{p}_{j}\right)^{\frac{m+1}{m-1}}}\right)^{\frac{m-1}{m}} .
$$

By the prime number theorem for $K$ and partial summation we infer that with $z:=\mathfrak{N p}^{+}(\mathfrak{m})$ the last sum is at most

$$
\leqslant \sum_{\mathfrak{N} \mathfrak{p}>z / 3} \frac{1}{\mathfrak{N p}(\log \mathfrak{N} \mathfrak{p})^{\frac{m+1}{m-1}}} \ll(\log z)^{-\frac{m+1}{m-1}}
$$

thus acquiring the validity of

$$
\mathscr{A}_{m}(\mathfrak{m}) \ll\left(\sum_{\substack{\mathfrak{p}_{j} \in \mathscr{P}_{K}^{\circ} \\ j>i^{+}(\mathfrak{m})}} \frac{W_{2 m}\left(\mathfrak{m}, \mathfrak{p}_{j}\right) \log \mathfrak{N} \mathfrak{p}_{j}}{\mathfrak{N p}_{j}}\right)^{\frac{1}{m}}(\log z)^{-\frac{m+1}{m-1}} .
$$

For $\vartheta \in \mathbb{R}$ and $\mathfrak{a} \in \mathscr{I}_{K}$ define

$$
\tau_{K}^{*}\left(\mathfrak{a} ; \psi_{K} ; \vartheta\right):=\sum_{\mathfrak{d} \mid \mathfrak{a}} \psi_{K}(\mathfrak{d}) \mathfrak{N} \mathfrak{d}^{i \vartheta} \quad \text { and } \quad \tau_{K}^{*}\left(\mathfrak{a} ; \psi_{K}\right):=\frac{1}{2 \pi} \int_{\mathbb{R}} \frac{\left|\tau_{K}^{*}\left(\mathfrak{a} ; \psi_{K} ; \vartheta\right)\right|^{2}}{6+\vartheta^{2}} \mathrm{~d} \vartheta
$$

Lemma 3.3. For all $\mathfrak{a} \in \mathscr{I}_{K}$ we have $M_{2}\left(\mathfrak{a} ; \psi_{K}\right) \leqslant \tau_{K}^{*}\left(\mathfrak{a} ; \psi_{K}\right)$.

Proof. We start by using the following well-known formula, valid for all $u, v, x \in \mathbb{R}$,

$$
-\frac{1}{2 \pi i} \int_{-\infty}^{+\infty} \frac{\mathrm{e}^{i t(x-v)}-\mathrm{e}^{i t(x-u)}}{t} \mathrm{~d} t= \begin{cases}1 & \text { if } u<x \text { and } x<v \\ 0 & \text { if } x<u \text { or } x>v\end{cases}
$$

a proof of which can be found, for example, in [Wie33, §5]. The substitution $t \mapsto 2 \pi r$ gives

$$
\Delta_{K}\left(\mathfrak{a} ; \psi_{K} ; a, b\right)=\int_{-\infty}^{+\infty}\left(\frac{1-\mathrm{e}^{-2 \pi i r b}}{2 \pi i r} \tau_{K}^{*}\left(\mathfrak{a}, \psi_{K} ; 2 \pi r\right)\right) \mathrm{e}^{-2 \pi i a r} \mathrm{~d} r
$$

except when $a, b$ assume a finite set of values, thus Plancherel's theorem leads to

$$
\int_{-\infty}^{+\infty} \Delta_{K}\left(\mathfrak{a} ; \psi_{K} ; a, b\right)^{2} \mathrm{~d} a=\frac{1}{2 \pi^{2}} \int_{-\infty}^{+\infty} \frac{1-\cos (2 \pi r b)}{r^{2}}\left|\tau_{K}^{*}\left(\mathfrak{a}, \psi_{K} ; 2 \pi r\right)\right|^{2} \mathrm{~d} r .
$$


It can then be inferred from $\int_{0}^{1}(1-\cos (2 \pi r b)) \mathrm{d} b=1-\frac{\sin (2 \pi r)}{2 \pi r}$ that $2 \pi^{2} M_{2}\left(\mathfrak{a} ; \psi_{K}\right)$ equals

$$
\int_{-\infty}^{+\infty}\left(1-\frac{\sin (2 \pi r)}{2 \pi r}\right) \frac{\left|\tau_{K}^{*}\left(\mathfrak{a}, \psi_{K} ; 2 \pi r\right)\right|^{2}}{r^{2}} \mathrm{~d} r
$$

and the inequality $1-\frac{\sin (2 \pi r)}{2 \pi r} \leqslant \frac{4 \pi^{2} r^{2}}{3+2 \pi^{2} r^{2}}$ furnishes the proof of our lemma.

Define the arithmetic function $g: \mathbb{N} \rightarrow \mathbb{Z}$ though $g(n):=\sharp\left\{\mathfrak{p} \subset \mathscr{O}_{K}: \mathfrak{N p}=n\right\}$ and note that the prime number theorem for $K$ provides a positive constant $\varkappa$ such that

$$
\sum_{1 \leqslant n \leqslant T} g(n)=\operatorname{li}(T)+O\left(T \mathrm{e}^{-(\log T)^{\varkappa}}\right) .
$$

Recall the definition of $M_{q}(\mathfrak{a})$ in (2.6) .

Lemma 3.4. For all $\Xi \geqslant 1$ and $m \in \mathbb{N}$ we have

$$
\begin{aligned}
m^{-1} 4^{-m} \sum_{\mathfrak{N} \mathfrak{p}>\Xi} \frac{W_{2 m}(\mathfrak{m}, \mathfrak{p}) \log \mathfrak{N \mathfrak { p }}}{\mathfrak{N p}} & \ll M_{2 m}\left(\mathfrak{m}, \psi_{K}\right)^{\frac{m-2}{m-1}} \tau_{K}^{*}\left(\mathfrak{m} ; \psi_{K}\right)^{\frac{m}{m-1}} \\
& +\mathrm{e}^{-(\log \Xi)^{\varkappa}} 4^{m} M_{2 m}(\mathfrak{m})^{\frac{2 m-2}{2 m-1}} \tau_{K}(\mathfrak{m})^{\frac{2 m}{2 m-1}}
\end{aligned}
$$

Proof. Using (3.13) shows that the sum in our lemma is bounded by

$$
\left(1+\frac{2}{3} m\right) \sum_{1 \leqslant j \leqslant m-1}\left(\begin{array}{c}
2 m \\
2 j
\end{array}\right) \sum_{\mathfrak{N} \mathfrak{p}>\Xi} \frac{N_{2 j, 2 m}(\mathfrak{m}, \log \mathfrak{N p}) \log \mathfrak{N p}}{\mathfrak{N p}}
$$

and the inner sum can be recast as

$$
\int_{0}^{1} \int_{\mathbb{R}} \Delta_{K}\left(\mathfrak{m} ; \psi_{K} ; a, b\right)^{2 j}\left(\sum_{\mathfrak{N} \mathfrak{p}>\Xi} \frac{\log \mathfrak{N \mathfrak { p }}}{\mathfrak{N p}} \Delta_{K}\left(\mathfrak{m} ; \psi_{K} ; a-\log \mathfrak{N \mathfrak { p }}, b\right)^{2 m-2 j}\right) \mathrm{d} a \mathrm{~d} b .
$$

Letting $h:=2 m-2 j$ allows us to see that the sum over $\mathfrak{p}$ equals

$$
\sum_{\substack{\mathfrak{d}_{1}, \ldots, \mathfrak{d}_{h} \in \mathscr{I}_{K} \\ \mathfrak{d}_{i} \mid \mathfrak{m}}} \psi_{K}\left(\mathfrak{d}_{1} \cdots \mathfrak{d}_{h}\right) \sum_{n>\Xi}^{*} g(n) \frac{\log n}{n}
$$

where the sum $\Sigma^{*}$ is over integers $n$ satisfying the further condition

$$
a-\min \log \mathfrak{N d}_{i}<\log n \leqslant a-\max \log \mathfrak{N d}_{i}+b .
$$

This implies that the sum contains no terms unless max $\mathfrak{N d}_{i}<\mathrm{e}^{b} \min \mathfrak{N o}_{i}$, in which case (3.17) and Abel's summation provide the bound

$$
\ll \int_{a-\min \log \mathfrak{N} \mathfrak{d}_{i}}^{a+b-\log \max \mathfrak{N} \mathfrak{d}_{i}} \mathbf{1}_{(0, \infty)}(t-\log \Xi) \mathrm{d} t+\mathrm{e}^{-(\log \Xi)^{\varkappa}},
$$

where $\mathbf{1}_{(0, \infty)}$ denotes the characteristic function of the positive real numbers. This confirms

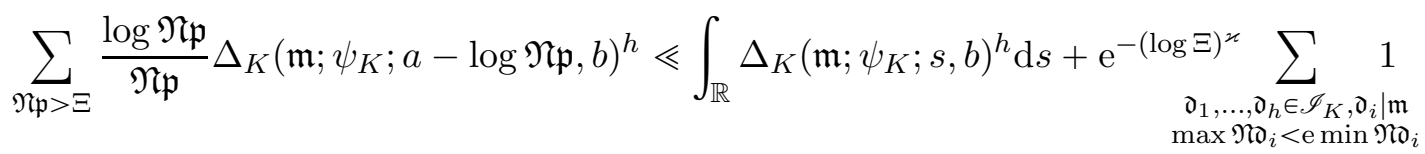


and according to Lemma 2.4 the inner sum is $\ll 2^{h} M_{h}(\mathfrak{m})$. Therefore the sum over the prime ideals in (3.18) is

$$
\begin{aligned}
& \ll \int_{0}^{1} \int_{\mathbb{R}} \Delta_{K}\left(\mathfrak{m} ; \psi_{K} ; a, b\right)^{2 j} \mathrm{~d} a \int_{\mathbb{R}} \Delta_{K}\left(\mathfrak{m} ; \psi_{K} ; s, b\right)^{2 m-2 j} \mathrm{~d} s \mathrm{~d} b \\
& +\mathrm{e}^{-(\log \Xi)^{\varkappa}} 4^{m-j} M_{2 j}(\mathfrak{m}) M_{2 m-2 j}(\mathfrak{m}),
\end{aligned}
$$

where we have used $\left|\psi_{K}(\mathfrak{d})\right| \leqslant 1$ to dispense with the integration over $0 \leqslant b \leqslant 1$ in the second term. In virtue of (2.7) and Lemma (3.3) one can show by following the argument involving Hölder's inequality at the end of the proof of [dIBT12, Lem. 2.4] that the last expression is

$$
\ll M_{2 m}\left(\mathfrak{m} ; \psi_{K}\right)^{\frac{m-2}{m-1}} \tau^{*}\left(\mathfrak{m} ; \psi_{K}\right)^{\frac{m}{m-1}}+\mathrm{e}^{-(\log \Xi)^{\varkappa}} 4^{m} M_{2 m}(\mathfrak{m})^{\frac{2 m-2}{2 m-1}} \tau_{K}(\mathfrak{m})^{\frac{2 m}{2 m-1}},
$$

which, in view of $\sum_{1 \leqslant j \leqslant m-1}\left(\begin{array}{c}2 m \\ 2 j\end{array}\right)=4^{m}-2$, finishes our proof.

We may now deploy the bound supplied by Lemma 3.4 in conjunction with (3.15) to obtain

$$
\mathscr{A}_{m}(\mathfrak{m}) \ll \mathscr{B}_{m}(\mathfrak{m})+\mathscr{C}_{m}(\mathfrak{m})
$$

where

$$
\mathscr{B}_{m}(\mathfrak{m}):=\frac{M_{2 m}\left(\mathfrak{m} ; \psi_{K}\right)^{\frac{m-2}{m(m-1)}} \tau_{K}^{*}\left(\mathfrak{m} ; \psi_{K}\right)^{\frac{1}{m-1}}}{(\log z)^{\frac{m+1}{m-1}}}
$$

and

$$
\mathscr{C}_{m}(\mathfrak{m}):=\frac{M_{2 m}(\mathfrak{m})^{\frac{2 m-2}{m(2 m-1)}} \tau_{K}(\mathfrak{m})^{\frac{2}{2 m-1}}}{\mathrm{e}^{\frac{1}{m}(\log z)^{\varkappa}}} .
$$

Alluding to Lemma 2.3, the term $\mathscr{C}_{m}(\mathfrak{m})$ makes the following contribution towards (3.14),

$$
\ll \frac{1}{\sigma} \sum_{\substack{\mathfrak{m} \in \mathscr{P}_{K}^{\circ} \\ \omega_{K}(\mathfrak{m})=t-1 \\ \mathfrak{p} \mid \mathfrak{m} \Rightarrow \mathfrak{N} \mathfrak{p}>z_{2}}} \frac{\mu_{K}^{2}(\mathfrak{m}) \tau_{K}(\mathfrak{m})^{2}}{\mathfrak{N m}^{1+\sigma}} \exp \left(-\frac{1}{m}\left(\log \mathfrak{N} \mathfrak{p}^{+}(\mathfrak{m})\right)^{\varkappa}\right) .
$$

Each $\mathfrak{m}$ above is the product of $\mathfrak{p}^{+}(\mathfrak{m})$ and $t-2$ prime ideals $\mathfrak{p}_{i}$ with $\mathfrak{N p}_{i} \leqslant \mathfrak{N p} \mathfrak{p}^{+}(\mathfrak{m})$. Taking into account the possible permutations of the ideals $\mathfrak{p}_{i}$ shows that the sum over $\mathfrak{m}$ is

$$
\ll \frac{1}{(t-2) !} \sum_{\mathfrak{N} \mathfrak{p}>z_{2}} \frac{\exp \left(-\frac{1}{m}(\log \mathfrak{N p})^{\varkappa}\right)}{\mathfrak{N p}}\left(\sum_{\mathfrak{T}_{\mathfrak{p}_{i} \leqslant \mathfrak{N} \mathfrak{p}}} \frac{4}{\mathfrak{N p}_{i}}\right)^{t-2}
$$

The sum over $\mathfrak{p}_{i}$ is at most $4 \log _{2} \mathfrak{N p}+O(1)$, hence using the inequality $\exp (-x) \leqslant \frac{\ell !}{x^{\ell}}$, valid for all $x \geqslant 0, \ell \in \mathbb{N}$, we obtain that the expression above is bounded by

$$
\ll \frac{4^{t} \ell ! m^{\ell}}{(t-1) !} \sum_{\mathfrak{p}>z_{2}} \frac{1}{\mathfrak{N p}} \frac{(\log \log \mathfrak{N p})^{t-2}}{(\log \mathfrak{N p})^{\varkappa \ell}} .
$$

We may suppose that $t^{\prime}$ satisfies $\left(\frac{x t^{\prime}}{5}-1\right)>1$ and $t^{\prime}>5$, so that upon choosing $\ell:=\left[\frac{t}{5}\right]$ we see that the sum is

$$
\ll \int_{z_{2}}^{\infty} \frac{(\log \log u)^{t}}{u(\log u)^{t / 5}} \mathrm{~d} u=\left(\frac{\varkappa t}{5}-1\right)^{-t} \int_{\left(\frac{\varkappa t}{5}-1\right) \log \log z_{2}}^{\infty} \frac{v^{t}}{\mathrm{e}^{v}} \mathrm{~d} v \leqslant\left(\frac{\varkappa t}{5}-1\right)^{-t} t ! .
$$


Therefore, using $\log m=\frac{1}{2} \log t+O(\log \log t)$ (which is implied by the assumptions of Proposition (3.2), as well as $\log n !=n \log n+O(n)$, we see that the contribution of the entity $\mathscr{C}_{m}(\mathfrak{m})$ towards (3.14) is

$$
\ll \sigma^{-1} \frac{4^{t} \ell ! m^{\ell} 10^{t}}{\lambda_{0}^{\ell} t^{t}} \leqslant \sigma^{-1} \exp \left(-\frac{7}{10} t \log t+O(t \log \log t)\right) \ll \frac{1}{\sigma(t !)^{2 / 3}} .
$$

We now turn our attention to the contribution of $\mathscr{B}_{m}(\mathfrak{m})$ to (3.14). It is at most

$$
\sum_{\substack{\mathfrak{m} \in \mathscr{P}_{K}^{\circ} \\ \omega_{K}(\mathfrak{m})=t-1 \\ \mathfrak{p} \mid \mathfrak{m} \Rightarrow \mathfrak{N} \mathfrak{p}>z_{2}}} \frac{M_{2 m}\left(\mathfrak{m} ; \psi_{K}\right)^{\frac{m-2}{m(m-1)}} \tau_{K}^{*}\left(\mathfrak{m} ; \psi_{K}\right)^{\frac{1}{m-1}}}{\left(\log \mathfrak{N} \mathfrak{p}^{+}(\mathfrak{m})\right)^{\frac{1}{m}}} \frac{\mu_{K}^{2}(\mathfrak{m})}{\mathfrak{N m}^{1+\sigma}} \frac{1}{\sigma \log \mathfrak{N} \mathfrak{p}^{+}(\mathfrak{m})} .
$$

For $\mathfrak{m}$ as in the sum above we let $S(\mathfrak{m})$ be the set of square-free elements $\mathfrak{n} \in \mathscr{P}_{K}^{\circ}$ that are divisible by $\mathfrak{m}$ with the further property that any prime ideal $\mathfrak{p}_{i} \mid \mathfrak{n}$ with $\mathfrak{p}_{i} \nmid \mathfrak{m}$ satisfies $i>i^{+}(\mathfrak{m})$. These ideals enjoy the property $\mathfrak{n}_{t-1}=\mathfrak{m}$ and therefore

$$
\sum_{\substack{\mathfrak{n} \in \mathscr{P}_{K}^{\circ} \\ \mathfrak{n}_{t-1}=\mathfrak{m}}} \frac{\mu_{K}(\mathfrak{n})^{2}}{\mathfrak{N n}^{1+\sigma}} \geqslant \sum_{\mathfrak{n} \in S(\mathfrak{m})} \frac{\mu_{K}(\mathfrak{n})^{2}}{\mathfrak{N n}^{1+\sigma}} \geqslant \frac{\mu_{K}(\mathfrak{m})^{2}}{\mathfrak{N m}^{1+\sigma}} \prod_{\substack{i>i^{+}(\mathfrak{m}) \\ \mathfrak{p}_{i} \in \mathscr{P}_{K}^{\circ}}}\left(1+\frac{1}{\mathfrak{N p}_{i}^{1+\sigma}}\right)
$$

The effect of primes $\mathfrak{p}_{i}$ with residue degree more than 1 is bounded by a constant depending only on $K$, thus the product is

$$
\gg \zeta_{K}(1+\sigma) \prod_{i \leqslant i^{+}(\mathfrak{m})}\left(1-\frac{1}{\mathfrak{N p}_{i}^{1+\sigma}}\right) \gg \frac{1}{\sigma} \exp \left(-\sum_{i \leqslant i^{+}(\mathfrak{m})} \frac{1}{\mathfrak{N p}^{1+\sigma}}\right),
$$

which by the Mertens theorem for $K$ is

$$
\gg \frac{1}{\sigma} \exp \left(-\sum_{i \leqslant i^{+}(\mathfrak{m})} \frac{1}{\mathfrak{N p}}\right) \gg \frac{1}{\sigma \log \mathfrak{N} \mathfrak{p}^{+}(\mathfrak{m})} .
$$

We deduce that the sum in (3.19) is

$$
\ll \sum_{\substack{\mathfrak{m} \in \mathscr{P}_{K}^{\circ} \\ \omega_{K}(\mathfrak{m})=t-1 \\ \mathfrak{p} \mid \mathfrak{m} \Rightarrow \mathfrak{N} \mathfrak{p}>z_{2}}} \frac{M_{2 m}\left(\mathfrak{m} ; \psi_{K}\right)^{\frac{m-2}{m(m-1)}} \tau_{K}^{*}\left(\mathfrak{m} ; \psi_{K}\right)^{\frac{1}{m-1}}}{\left(\log \mathfrak{N} \mathfrak{p}^{+}(\mathfrak{m})\right)^{\frac{1}{m}}} \sum_{\substack{\mathfrak{n} \in \mathscr{P}_{K}^{\circ} \\ \mathfrak{n} t-1}} \frac{\mu_{K}(\mathfrak{n})^{2}}{\mathfrak{N n}^{1+\sigma}} .
$$

Observe that for each $\mathfrak{n}$ in the inner sum we have $\omega_{K}(\mathfrak{n}) \geqslant \omega_{K}(\mathfrak{m})=t-1$ and therefore the double sum may be reshaped into

$$
\sum_{\substack{\mathfrak{n} \in \mathscr{P}_{K}^{\circ} \\ \omega_{K}(\mathfrak{n}) \geqslant t-1}}\left(\frac{\mu_{K}(\mathfrak{n})^{2} M_{2 m}\left(\mathfrak{n}_{t-1} ; \psi_{K}\right)^{\frac{m-2}{m(m-1)}}}{\mathfrak{N n}^{\frac{(1+\sigma)(m-2)}{m-1}}}\right)\left(\frac{\mu_{K}(\mathfrak{n})^{2} \tau_{K}^{*}\left(\mathfrak{n}_{t-1} ; \psi_{K}\right)^{\frac{1}{m-1}}}{\mathfrak{N n}^{\frac{1+\sigma}{m-1}}\left(\log \mathfrak{N}^{+}\left(\mathfrak{n}_{k}\right)\right)^{\frac{1}{m}}}\right) .
$$

Assuming that $t^{\prime}$ is large enough so that $m>2$ we can gain the succeeding bound via a use of Hölder's inequality with exponents $\frac{m-1}{m-2}, m-1$,

$$
\ll L_{t-1, m}^{*}(\sigma)^{\frac{m-2}{m-1}} \mathscr{D}_{t-1, m}(\sigma)^{\frac{1}{m-1}},
$$


where for $t, m$ positive integers and $\hat{\sigma} \in\left(0, \frac{1}{4}\right)$ we have defined

$$
\mathscr{D}_{t-1, m}(\widehat{\sigma}):=\sum_{\substack{\mathfrak{n} \in \mathscr{P}_{K}^{\circ} \\ \omega_{K}(\mathfrak{n}) \geqslant t-1}} \frac{\mu_{K}(\mathfrak{n})^{2} \tau_{K}^{*}\left(\mathfrak{n}_{k} ; \psi\right)}{\mathfrak{N n}^{1+\hat{\sigma}}\left(\log \mathfrak{N p}^{+}\left(\mathfrak{n}_{t-1}\right)\right)^{\frac{m-1}{m}}}
$$

To estimate $\mathscr{D}_{t-1, m}(\widehat{\sigma})$ we shall need the following lemma.

Lemma 3.5. For $\vartheta, \Gamma \in(0, \infty)$ define

$$
S(\Gamma ; \vartheta):=\sum_{\substack{\mathfrak{N} \mathfrak{p} \leqslant \Gamma \\ \mathfrak{p} \in \mathscr{P}_{K}^{\circ}}} \frac{\left|1+\psi_{K}(\mathfrak{p}) \mathfrak{N p}^{i \vartheta}\right|^{2}}{\mathfrak{N p}}
$$

There exists a constant $B=B\left(K, \psi_{K}\right)$ such that the following holds uniformly in $\vartheta$,

$$
S(\Gamma ; \vartheta) \leqslant \begin{cases}2 \log (1+|\vartheta| \log \Gamma)+2 \log \left(\frac{\log \Gamma}{1+|\vartheta| \log \Gamma}\right)+O(1), & \text { if } 0<|\vartheta| \leqslant 1 \\ 2 \log \log \Gamma+B \log \log (2+|\vartheta|), & \text { otherwise, }\end{cases}
$$

where the implied constants are independent of $\Gamma$ and $\vartheta$.

Proof. For $j=1,-1$ we let

$$
S_{j}(\Gamma ; \vartheta):=\sum_{\substack{\mathfrak{N} \mathfrak{p} \leqslant \Gamma \\ \mathfrak{p} \in \mathscr{P}_{K}^{\circ}, \psi_{K}(\mathfrak{p})=j}} \frac{\left|1+\psi_{K}(\mathfrak{p}) \mathfrak{N p}^{i \vartheta}\right|^{2}}{\mathfrak{N p}}
$$

so that $S(\Gamma ; \vartheta)=\sum_{j \in\{1,-1\}} S_{j}(\Gamma ; \vartheta)+O(1)$. Introduce the functions $g_{j}: \mathbb{N} \rightarrow \mathbb{Z}_{\geqslant 0}$ via

$$
g_{j}(n):=\sharp\left\{\mathfrak{a} \in \mathscr{P}_{K}^{\circ}: \mathfrak{N a}=n, \psi_{K}(\mathfrak{a})=j\right\}
$$

and note that the condition $\mathfrak{p} \in \mathscr{P}_{K}^{\circ}$ forces $\mathfrak{N p}$ to be a rational prime. The quantitative version of Chebotarev's theorem provides positive constants $c^{\prime}, \eta^{\prime}$ such that

$$
\sum_{p \leqslant \Gamma} g_{j}(p)=\sum_{\substack{\mathfrak{N} \mathfrak{p} \leqslant \Gamma \\ \mathfrak{p} \in \mathscr{P}_{K}^{\circ}, \psi_{K}(\mathfrak{p})=j}} 1=\frac{\operatorname{li}(\Gamma)}{2}+O\left(\Gamma \mathrm{e}^{-c^{\prime}(\log \Gamma)^{\eta^{\prime}}}\right),
$$

due to the standard bound

$$
\sum_{\substack{\mathfrak{N} \mathfrak{p} \leqslant \Gamma \\ f_{\mathfrak{p}}>1}} 1 \ll_{\varepsilon, K} \Gamma^{\frac{1}{2}+\varepsilon}
$$

valid for all $\varepsilon>0$. Hence, in the notation of [Ten15, Lem.III.4.13], we can use $h(r):=\left|1+\mathrm{e}^{i r}\right|^{2}$ and directly modify its proof to show that for each $w<\Gamma$ the following equality holds uniformly in $\vartheta \neq 0$,

$$
\sum_{\substack{w<\mathfrak{N} \mathfrak{p} \leqslant \Gamma \\ \mathfrak{p} \in \mathscr{P}_{K}^{\circ} \\ \psi_{K}(\mathfrak{p})=j}} \frac{\left|1+j \mathfrak{N p}^{i \vartheta}\right|^{2}}{\mathfrak{N p}}=\sum_{w<p \leqslant \Gamma}\left|1+j p^{i \vartheta}\right|^{2} \frac{g_{j}(p)}{p}=\log \left(\frac{\log \Gamma}{\log w}\right)+O\left(\frac{1}{|\vartheta| \log w}+\frac{1+|\vartheta|}{\mathrm{e}^{c^{\prime \prime}(\log w)^{\eta^{\prime}}}}\right),
$$

owing to $\bar{h}=2$ for our choice of $h$. This equality is parallel to [dBT12, Eq.(3.16)], our proof can thus be concluded as the one of [dlBT12, Lem.2.5] by using it for suitable parameters $w$ according to the value of $\vartheta$ in relation to $\Gamma$. 
Lemma 3.6. For all $t, m$ as in Proposition 3.2 and $\hat{\sigma} \in \mathbb{R} \cap\left(0, \frac{1}{4}\right)$, we have

$$
\mathscr{D}_{t-1, m}(\hat{\sigma}) \ll \frac{t-1}{\hat{\sigma}\left(1-\frac{1}{2 m}\right)^{t-1}} .
$$

Proof. Fix an element $\mathfrak{m} \in \mathscr{P}_{K}^{\circ}$. The integral ideals $\mathfrak{n}$ in $\mathscr{D}_{t-1, m}(\widehat{\sigma})$ with $\mathfrak{n}_{t-1}=\mathfrak{m}$ are of the shape $\mathfrak{n}=\mathfrak{m} \mathfrak{d}$, where $\mathfrak{d}$ is square-free and furthermore if $\mathfrak{p}_{i} \mid \mathfrak{d}$ then $i>i^{+}(\mathfrak{m})$. Hence,

$$
\mathscr{D}_{t-1, m}(\widehat{\sigma}) \leqslant \sum_{\substack{\mathfrak{m} \in \mathscr{P}_{K}^{\circ} \\ \omega_{K}(\mathfrak{m})=t-1}} \frac{\mu_{K}(\mathfrak{m})^{2} \tau_{K}^{*}\left(\mathfrak{m} ; \psi_{K}\right)}{\mathfrak{N m}^{1+\hat{\sigma}}\left(\log \mathfrak{N p}^{+}(\mathfrak{m})\right)^{\frac{m-1}{m}}} \prod_{i>i^{+}(\mathfrak{m})}\left(1+\frac{1}{\mathfrak{N p}_{i}^{1+\hat{\sigma}}}\right)
$$

The last product is

$$
\ll \zeta_{K}(1+\widehat{\sigma}) \exp \left(-\sum_{i \leqslant i^{+}(\mathfrak{m})} \mathfrak{N p}_{i}^{-1-\hat{\sigma}}\right) \ll \hat{\sigma}^{-1} \exp \left(-\sum_{i \leqslant i^{+}(\mathfrak{m})} \mathfrak{N p}_{i}^{-1-\hat{\sigma}}\right) .
$$

The inequality $1-\mathfrak{N p}_{i}^{-\widehat{\sigma}} \leqslant \widehat{\sigma} \log \mathfrak{N p}_{i}$ reveals that

$$
\sum_{i \leqslant i^{+}(\mathfrak{m})} \mathfrak{N p}_{i}^{-1-\hat{\sigma}} \geqslant \sum_{i \leqslant i^{+}(\mathfrak{m})} \frac{1}{\mathfrak{N p}_{i}}-\hat{\sigma} \sum_{i \leqslant i^{+}(\mathfrak{m})} \frac{\log \mathfrak{N p}_{i}}{\mathfrak{N p}_{i}}
$$

which, by the prime number theorem for $K$, is $\ll \log \log \mathfrak{N p}^{+}(\mathfrak{m})-\hat{\sigma} \log \mathfrak{N p}^{+}(\mathfrak{m})$. We can therefore bound $\mathscr{D}_{t-1, m}(\widehat{\sigma})$ by

$$
\ll \frac{1}{\widehat{\sigma}} \sum_{\substack{\mathfrak{m} \in \mathscr{P}_{K}^{\circ} \\ \omega_{K}(\mathfrak{m})=t-1}} \frac{\mu_{K}(\mathfrak{m})^{2} \tau_{K}^{*}\left(\mathfrak{m} ; \psi_{K}\right)}{\mathfrak{N m}(\log \mathfrak{N} \mathfrak{p}+(\mathfrak{m}))^{\frac{2 m-1}{m}}} \frac{\mathfrak{N p}^{+}(\mathfrak{m})^{\hat{\sigma}}}{\mathfrak{N m} \hat{w}^{\hat{\sigma}}}
$$

and, letting

$$
T:=\sum_{\substack{\mathfrak{m} \in \mathscr{P}_{K}^{\circ} \\ \omega_{K}(\mathfrak{m})=t-1}} \frac{\mu_{K}(\mathfrak{m})^{2} \tau_{K}^{*}\left(\mathfrak{m} ; \psi_{K}\right)}{\mathfrak{N m}\left(\log \mathfrak{N p} \mathfrak{p}^{+}(\mathfrak{m})\right)^{\frac{2 m-1}{m}}}
$$

allows us to deploy the inequality $\mathfrak{N p}^{+}(\mathfrak{m}) \leqslant \mathfrak{N m}$ to infer that $\mathscr{D}_{t-1, m}(\widehat{\sigma}) \ll T / \widehat{\sigma}$. For $\vartheta \in \mathbb{R}$ let

$$
T(\vartheta):=\sum_{\substack{\mathfrak{m} \subset \mathscr{P}_{K}^{\circ} \\ \omega_{K}(\mathfrak{m})=t-1}} \frac{\mu_{K}(\mathfrak{m})^{2}\left|\tau_{K}^{*}\left(\mathfrak{a} ; \psi_{K} ; \vartheta\right)\right|^{2}}{\mathfrak{N m}\left(\log \mathfrak{N} \mathfrak{p}^{+}(\mathfrak{m})\right)^{\frac{2 m-1}{m}}} .
$$

Note that alluding to $(\underline{3.16)})$ and using $\tau^{*}(\mathfrak{m}, \psi ;-\vartheta)=\overline{\tau^{*}(\mathfrak{m}, \psi ; \vartheta)}$ provides us with

$$
T \ll \int_{0}^{\infty} T(\vartheta)\left(1+\vartheta^{2}\right)^{-1} \mathrm{~d} \vartheta
$$

Denote $\mathfrak{p}^{\prime}=\mathfrak{p}^{+}(\mathfrak{m})$. Each ideal $\mathfrak{m}$ in $T(\vartheta)$ is the product of $\mathfrak{p}^{\prime}$ and $k-1$ different prime ideals $\mathfrak{p}_{i} \in \mathscr{P}_{K}^{\circ}$ that satisfy $i<i^{+}(\mathfrak{m})$. Therefore

$$
T(\vartheta) \ll \frac{1}{(t-2) !} \sum_{\mathfrak{p}^{\prime} \in \mathscr{P}{ }_{K}^{\circ}} \frac{\left|\tau_{K}^{*}\left(\mathfrak{p}^{\prime} ; \psi ; \vartheta\right)\right|^{2}}{\mathfrak{N} \mathfrak{p}^{\prime}\left(\log \mathfrak{N} \mathfrak{p}^{\prime}\right)^{\frac{2 m-1}{m}}}\left(\sum_{\substack{\mathfrak{N} \mathfrak{p} \leqslant \mathfrak{N} \mathfrak{p}^{\prime} \\ \mathfrak{p} \in \mathscr{P} \%}} \frac{\left|\tau_{K}^{*}\left(\mathfrak{p} ; \psi_{K} ; \vartheta\right)\right|^{2}}{\mathfrak{N} \mathfrak{p}}\right)^{t-2}
$$


and using Lemma 3.5 allows us to follow the arguments proving [1BT12, Eq.(2.25),(2.26)] to acquire the bound

$$
\int_{0}^{1} T(\vartheta) \mathrm{d} \vartheta \ll \frac{2^{t}}{(t-2) !}\left\{\frac{(t-2) !}{\left(\frac{2 m-1}{m}+1\right)^{t-1}}+\frac{(t-1) !}{\left(\frac{2 m-1}{m}\right)^{t-1}}+\frac{(t-1) !}{\left(\frac{2 m-1}{m}+1\right)^{t-1}}\right\} \ll \frac{t-1}{\left(1-\frac{1}{2 m}\right)^{t-1}} .
$$

In the remaining range, $\vartheta>1$, one can conjure up Lemma 3.5] and the proof of [lBT12, Eq.(2.27)] to deduce the estimate

$$
T(\vartheta) \ll\left(1-\frac{1}{2 m}\right)^{-(t-1)}\{\log (2+\vartheta)\}^{\frac{B}{1-\frac{1}{2 m}}}
$$

which, in light of

is sufficient for our lemma.

$$
\int_{1}^{\infty}\{\log (2+\vartheta)\}^{\frac{B}{1-\frac{1}{2 m}}} \vartheta^{-2} \mathrm{~d} \vartheta \ll_{B} 1
$$

Assorting all appropriate estimates obtained so far validates

$$
L_{t, m}^{*}(\sigma)-4^{\frac{1}{m}} L_{t-1, m}^{*}(\sigma) \ll \frac{L_{t-1, q}^{*}(\sigma)^{\frac{m-2}{m-1}}(t-1)^{\frac{1}{m-1}}}{\sigma^{\frac{1}{m-1}}\left(1-\frac{1}{2 m}\right)^{\frac{t-1}{m-1}}}+\frac{1}{\sigma(t !)^{2 / 3}} .
$$

Bringing into play the entity

and noting that

$$
L_{k, q}^{*}(\sigma):=L_{k, q}(\sigma)+\frac{4^{\frac{k}{q}}}{\sigma}
$$

$$
L_{k+1, q}(\sigma)-4^{\frac{1}{q}} L_{k, q}(\sigma)=L_{k+1, q}^{*}(\sigma)-4^{\frac{1}{q}} L_{k, q}^{*}(\sigma), \frac{4^{\frac{t-1}{m}}}{\sigma} \leqslant L_{t-1, m}^{*}(\sigma)
$$

allows to gain via (3.20) the following inequality,

$$
L_{t, m}^{*}(\sigma) \leqslant L_{t-1, m}^{*}(\sigma)\left(4^{\frac{1}{m}}+\frac{(t-1)^{\frac{1}{m-1}}}{4^{\frac{t}{m(m-1)}}\left(1-\frac{1}{2 m}\right)^{\frac{t-1}{m-1}}}+\frac{1}{4^{\frac{t-1}{m}}(t !)^{2 / 3}}\right) .
$$

Using the fact $m=f(t)+O(1)$ shows that the middle term in the parenthesis is

$$
\frac{1}{t^{\log 4-\frac{1}{2}+o(1)}} \text {. }
$$

Hence, there exists $c_{3}>0$, depending at most on $K$ and $\psi_{K}$ such that

$$
L_{t, m}^{*}(\sigma) \leqslant \mathrm{e}^{\frac{c_{3}}{m}} L_{t-1, m}^{*}(\sigma),
$$

an estimate that concludes the proof of Proposition 3.2 .

\section{REFERENCES}

[Brü12] J. Brüdern, Daniel's twists of Hooley's delta function, Contributions in analytic and algebraic number theory, Springer Proc. Math., vol. 9, Springer, New York, 2012, pp. 31-82.

[BS16] T. Browning and E. Sofos, Counting rational points on quartic del Pezzo surfaces with a rational conic, Submitted (2016).

[dlBT12] R. de la Bretèche and G. Tenenbaum, Oscillations localisées sur les diviseurs, J. Lond. Math. Soc. (2) 85 (2012), no. 3, 669-693.

[dlBT13] _ Sur la conjecture de Manin pour certaines surfaces de Châtelet, J. Inst. Math. Jussieu 12 (2013), no. 4, 759-819. 
[dlBT16] _ Private communication.

[FMT89] J. Franke, Y. I. Manin, and Y. Tschinkel, Rational points of bounded height on Fano varieties, Invent. Math. 95 (1989), no. 2, 421-435.

[Hoo79] C. Hooley, On a new technique and its applications to the theory of numbers, Proc. London Math. Soc. (3) 38 (1979), no. 1, 115-151.

[NT98] M. Nair and G. Tenenbaum, Short sums of certain arithmetic functions, Acta Math. 180 (1998), no. 1, 119-144.

[Shi80] P. Shiu, A Brun-Titchmarsh theorem for multiplicative functions, J. reine angew. Math. 313 (1980), 161-170.

[Ten85] G. Tenenbaum, Sur la concentration moyenne des diviseurs, Comment. Math. Helv. 60 (1985), no. 3, 411-428.

[Ten86] Fonctions $\Delta$ de Hooley et applications, Séminaire de théorie des nombres, Paris 1984-85, Progr. Math., vol. 63, Birkhäuser Boston, Boston, MA, 1986, pp. 225-239.

[Ten90] _ Sur une question d'Erdös et Schinzel. II, Invent. Math. 99 (1990), no. 1, $215-224$.

[Ten15] - Introduction to analytic and probabilistic number theory, third ed., Graduate Studies in Mathematics, vol. 163, American Mathematical Society, Providence, RI, 2015.

[Vau85] R. C. Vaughan, Sur le problème de Waring pour les cubes, C. R. Acad. Sci. Paris Sér. I Math. 301 (1985), no. 6, 253-255.

[Vau86] - On Waring's problem for cubes, J. reine angew. Math. 365 (1986), 122-170.

[Wie33] N. Wiener, The Fourier integral and certain of its applications, Cambridge Mathematical Library, Cambridge University Press, Cambridge, 1933.

Max Planck Institute for Mathematics, Vivatsgasse 7, Bonn, 53111, Germany

E-mail address: sofos@mpim-bonn.mpg.de 